\title{
An Existence Theory for Gravity-Capillary Solitary Water Waves
}

\author{
M. D. Groves ${ }^{1}$
}

Received: 8 December 2019 / Accepted: 27 October 2020 / Published online: 16 February 2021

(C) The Author(s) 2021

\begin{abstract}
In the applied mathematics literature solitary gravity-capillary water waves are modelled by approximating the standard governing equations for water waves by a Korteweg-de Vries equation (for strong surface tension) or a nonlinear Schrödinger equation (for weak surface tension). These formal arguments have been justified by sophisticated techniques such as spatial dynamics and centre-manifold reduction methods on the one hand and variational methods on the other. This article presents a complete, self-contained account of an alternative, simpler approach in which one works directly with the Zakharov-Craig-Sulem formulation of the water-wave problem and uses only rudimentary fixed-point arguments and Fourier analysis.
\end{abstract}

Keywords Solitary waves · Dirichlet-Neumann operator $\cdot$ Korteweg-de Vries equation $\cdot$ Nonlinear Schrödinger equation

\section{Introduction}

\subsection{The Main Results}

The classical water-wave problem concerns the two-dimensional, irrotational flow of a perfect fluid of unit density subject to the forces of gravity and surface tension. We use dimensionless variables, choosing $h$ as length scale, $(h / g)^{\frac{1}{2}}$ as time scale and introducing the Bond number $\beta=\sigma / g h^{2}$, where $h$ is the depth of the water in its undisturbed state, $g$ is the acceleration due to gravity and $\sigma>0$ is the coefficient of surface tension. The fluid thus occupies the domain $D_{\eta}=\{(x, y): x \in \mathbb{R}$, $y \in(0,1+\eta(x, t))\}$, where $(x, y)$ are the usual Cartesian coordinates and $\eta>-1$ is a function of the spatial coordinate $x$ and time $t$, and the mathematical problem is

In memory of Walter Craig.

M. D. Groves

groves@math.uni-sb.de

1 Fachrichtung Mathematik, Universität des Saarlandes, Postfach 151150, 66041 Saarbrücken, Germany 
formulated in terms of an Eulerian velocity potential $\varphi(x, y, t)$ which solves Laplace's equation

$$
\varphi_{x x}+\varphi_{y y}=0, \quad 0<y<1+\eta,
$$

and the boundary conditions

$$
\begin{array}{ll}
\varphi_{y}=0, & y=0, \\
\eta_{t}=\varphi_{y}-\eta_{x} \varphi_{x}, & y=1+\eta, \\
\varphi_{t}=-\frac{1}{2} \varphi_{x}^{2}-\frac{1}{2} \varphi_{y}^{2}-\eta+\beta\left[\frac{\eta_{x}}{\sqrt{1+\eta_{x}^{2}}}\right]_{x}, & y=1+\eta
\end{array}
$$

Travelling waves are solutions of (1.1)-(1.4) of the form $\eta(x, t)=\eta(x-c t)$, $\varphi(x, y, t)=\varphi(x-c t, y)$, while solitary waves are non-trivial travelling waves which satisfy the asymptotic conditions $\eta(x-c t) \rightarrow 0$ as $|x-c t| \rightarrow \infty$; they correspond to localised disturbances of permanent form which move from left to right with constant speed $c$.

It is instructive to review the formal weakly nonlinear theory for travelling waves. We begin with the linear dispersion relation for a two-dimensional periodic travelling wave train of wave number $k \geq 0$ and speed $c>0$, namely

$$
c^{2}=\frac{1+\beta k^{2}}{f(k)}, \quad f(k)=k \operatorname{coth} k
$$

(see Fig. 1). The function $k \mapsto c(k)$ has a unique global minimum at $k=\omega$, and one finds that $\omega=0$ (with $c(0)=1$ ) for $\beta>\frac{1}{3}$ and $\omega>0$ for $\beta<\frac{1}{3}$. We denote the minimum value of $c$ by $c_{0}$, so that $c_{0}^{2}=1$ for $\beta>\frac{1}{3}$ and $c_{0}^{2}=2 \omega /\left(2 \omega f(\omega)-\omega^{2} f^{\prime}(\omega)\right)$ for $\beta<\frac{1}{3}$ (the formula $\beta=f^{\prime}(\omega) /\left(2 \omega f(\omega)-\omega^{2} f^{\prime}(\omega)\right)$ defines a bijection between the values of $\beta \in\left(0, \frac{1}{3}\right)$ and $\left.\omega \in(0, \infty)\right)$. Using $c$ as a bifurcation parameter, we expect branches of small-amplitude solitary waves to bifurcate at $c=c_{0}$ (where the
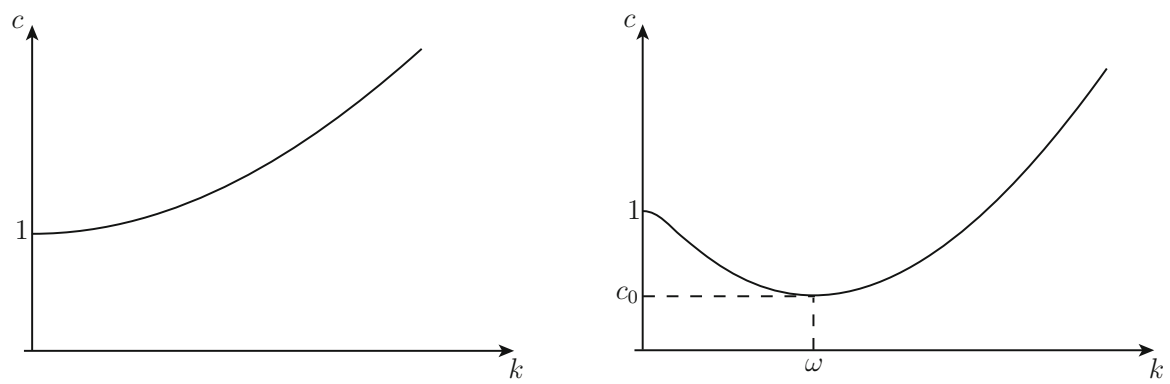

Fig. 1 Dispersion relation for a travelling wave train of wave number $k \geq 0$ and speed $c>0$ with strong surface tension (left) and weak surface tension (right) 


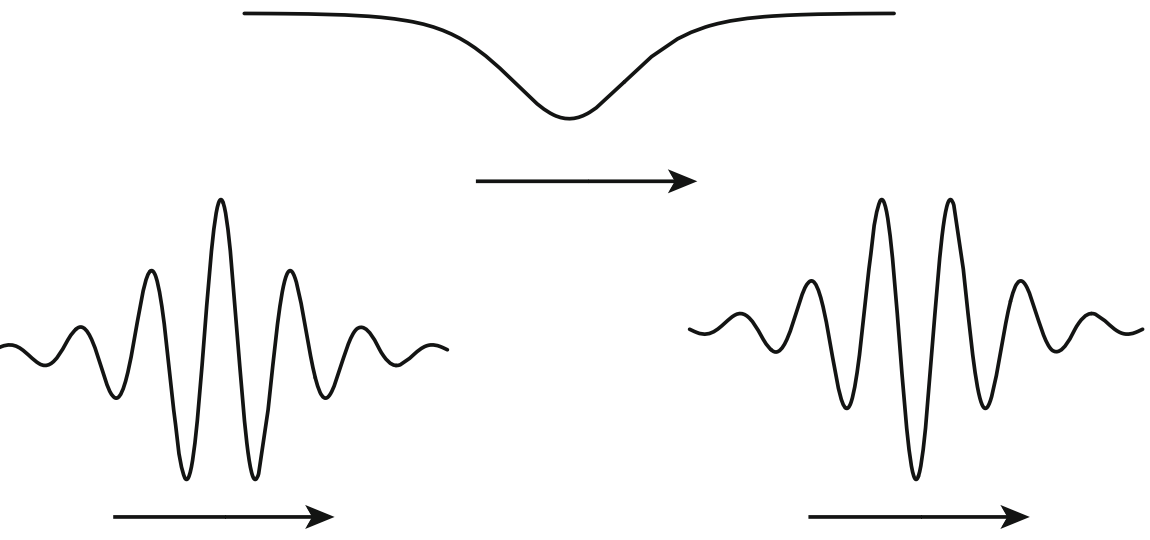

Fig. 2 Solitary wave of depression predicted by the Korteweg-de Vries equation for strong surface tension (above) and the symmetric solitary waves predicted by the nonlinear Schrödinger equation for weak surface tension (below)

linear group and phase speeds are equal) into the region $\left\{c<c_{0}\right\}$ where linear periodic wave trains are not supported (see Dias and Kharif [8, Sect. 3]).

In the case $\beta>\frac{1}{3}$ ('strong surface tension'), one writes $c^{2}=1-\varepsilon^{2}$, where $\varepsilon$ is a small positive number, substitutes the Ansatz

$$
\eta(x)=\varepsilon^{2} \rho_{1}(X)+\varepsilon^{2} \rho_{2}(X)+\cdots,
$$

where $X=\varepsilon x$, into the travelling-wave version of Eqs. (1.1)-(1.4), and finds that $\rho_{1}$ satisfies the stationary Korteweg-de Vries equation

$$
\rho-\left(\beta-\frac{1}{3}\right) \rho_{X X}+\frac{3}{2} \rho^{2}=0
$$

this equation admits an explicit solitary wave of depression given by the formula

$$
\rho^{\star}(X)=-\operatorname{sech}^{2}\left(\frac{X}{2\left(\beta-\frac{1}{3}\right)^{1 / 2}}\right)
$$

(see Benjamin [3]). In the case $\beta<\frac{1}{3}$ ('weak surface tension'), one writes $c^{2}=c_{0}^{2}\left(1-\varepsilon^{2}\right)$, uses the Ansatz

$$
\begin{aligned}
\eta(x)= & \frac{1}{2} \varepsilon\left(\zeta_{1}(X) \mathrm{e}^{\mathrm{i} \omega x}+\overline{\zeta_{1}(X)} \mathrm{e}^{-\mathrm{i} \omega x}\right)+\varepsilon^{2} \zeta_{0}(X) \\
& +\frac{1}{2} \varepsilon^{2}\left(\zeta_{2}(X) \mathrm{e}^{2 \mathrm{i} \omega x}+\overline{\zeta_{2}(X)} \mathrm{e}^{-2 \mathrm{i} \omega x}\right)+\cdots,
\end{aligned}
$$

and finds that $\zeta_{1}$ satisfies the stationary nonlinear Schrödinger equation

$$
-a_{1} \zeta_{X X}+a_{2} \zeta-a_{3}|\zeta|^{2} \zeta=0
$$


where

$$
a_{1}=\frac{1}{2} g^{\prime \prime}(\omega), \quad a_{2}=c_{0}^{2} f(\omega)
$$

and

$$
a_{3}=\frac{1}{4}\left(2 g(2 \omega)^{-1} A(\omega)^{2} c_{0}^{4}+2 g(0)^{-1} B(\omega)^{2} c_{0}^{4}+C(\omega) c_{0}^{2}+\frac{3}{2} \beta \omega^{4}\right)
$$

with

$$
\begin{aligned}
& A(\omega)=\frac{3}{2} \omega^{2}-\frac{1}{2} f(\omega)^{2}-f(\omega) f(2 \omega), \quad B(\omega)=\omega^{2}-2 f(\omega)-f(\omega)^{2}, \\
& C(\omega)=-6 \omega^{2} f(\omega)+4 f(\omega)^{2}+2 f(\omega)^{2} f(2 \omega)
\end{aligned}
$$

and

$$
g(k)=1+\beta k^{2}-c_{0}^{2} f(k)
$$

(see Ablowitz and Segur [1]). This equation admits a family $\left\{\mathrm{e}^{\mathrm{i} \theta_{0}} \zeta^{\star}\right\}_{\theta_{0} \in[0,2 \pi)}$ of solitary-wave solutions, where

$$
\zeta^{\star}(X)=\left(\frac{2 a_{2}}{a_{3}}\right)^{1 / 2} \operatorname{sech}\left(\frac{a_{2}}{a_{1}}\right)^{1 / 2} X,
$$

two of which, namely $\pm \zeta^{\star}$ (corresponding to $\theta_{0}=0$ and $\pi$ ), are symmetric. (The positivity of $a_{3}$ follows by elementary arguments after substituting the expressions for $\beta$ and $c_{0}^{2}$ as functions of $\omega$.) The corresponding free-surface profiles are sketched in Fig. 2.

The results of these formal calculations have been rigorously confirmed by spatial dynamics and centre-manifold methods on the one hand (Kirchgässner [17], Amick and Kirchgässner [2], Sachs [20], Iooss and Kirchgässner [15], Iooss and Pérouème [16]) and variational techniques on the other (Buffoni [4,5], Groves and Wahlén [11, 12]), the results of which are summarised in the following theorem.

\section{Theorem 1.1}

(i) Suppose that $\beta>\frac{1}{3}$ and $c^{2}=1-\varepsilon^{2}$. For each sufficiently small value of $\varepsilon>0$ there exists a symmetric solitary-wave solution of (1.1)-(1.4) whose free surface is given by

$$
\eta(x)=\varepsilon^{2} \rho^{\star}(\varepsilon x)+o\left(\varepsilon^{2}\right)
$$

uniformly over $x \in \mathbb{R}$.

(ii) Suppose that $\beta<\frac{1}{3}$ and $c^{2}=c_{0}^{2}\left(1-\varepsilon^{2}\right)$, where $c_{0}=c(\omega)$ is the global minimum of the linear dispersion relation (see Fig. 1 (right)). For each sufficiently small 
value of $\varepsilon>0$ there exist two symmetric solitary-wave solutions of (1.1)-(1.4) whose free surfaces are given by

$$
\eta(x)= \pm \varepsilon \zeta^{\star}(\varepsilon x) \cos \omega x+o(\varepsilon)
$$

uniformly over $x \in \mathbb{R}$.

This article presents an alternative, simpler proof of Theorem 1.1 in which one works directly with the Zakharov-Craig-Sulem formulation of the travelling waterwave equations (see below) and uses only rudimentary fixed-point arguments and Fourier analysis. Some intermediate results are special cases of more general theorems available elsewhere; their proofs have been included here for the sake of a complete, self-contained exposition.

\subsection{Methodology}

We proceed by formulating the water-wave problem (1.1)-(1.4) in terms of the variables $\eta$ and $\Phi=\left.\varphi\right|_{y=1+\eta}$ (see Zakharov [22] and Craig and Sulem [7]). The Zakharov-Craig-Sulem formulation of the water-wave problem is

$$
\begin{aligned}
& \eta_{t}-G(\eta) \Phi=0 \\
& \Phi_{t}+\eta+\frac{1}{2} \Phi_{x}^{2}-\frac{\left(G(\eta) \Phi+\eta_{x} \Phi_{x}\right)^{2}}{2\left(1+\eta_{x}^{2}\right)}-\beta\left[\frac{\eta_{x}}{\sqrt{1+\eta_{x}^{2}}}\right]_{x}=0
\end{aligned}
$$

where the velocity potential $\varphi$ is recovered as the (unique) solution of the boundaryvalue problem

$$
\begin{array}{ll}
\varphi_{x x}+\varphi_{y y}=0, & 0<y<1+\eta, \\
\varphi_{y}=0, & y=0, \\
\varphi=\Phi, & y=1+\eta,
\end{array}
$$

and the Dirichlet-Neumann operator $G(\eta)$ is given by $G(\eta) \Phi=\varphi_{y}-\left.\eta_{x} \varphi_{x}\right|_{y=1+\eta}$. Travelling waves are solutions of the form $\eta(x, t)=\eta(x-c t), \Phi(x, t)=\Phi(x-c t)$; they satisfy

$$
\begin{aligned}
& -c \eta_{x}-G(\eta) \Phi=0 \\
& -c \Phi_{x}+\eta+\frac{1}{2} \Phi_{x}^{2}-\frac{\left(G(\eta) \Phi+\eta_{x} \Phi_{x}\right)^{2}}{2\left(1+\eta_{x}^{2}\right)}-\beta\left[\frac{\eta_{x}}{\sqrt{1+\eta_{x}^{2}}}\right]_{x}=0
\end{aligned}
$$

It is possible to reduce Eqs. (1.11), (1.12) to a single equation for $\eta$. Using (1.11), one finds that $\Phi=-c G(\eta)^{-1} \eta_{x}$, and inserting this formula into (1.12) yields the equation

$$
\mathcal{K}(\eta)-c^{2} \mathcal{L}(\eta)=0
$$


where

$$
\begin{aligned}
& \mathcal{K}(\eta)=\eta-\beta\left[\frac{\eta_{x}}{\sqrt{1+\eta_{x}^{2}}}\right]_{x}, \\
& \mathcal{L}(\eta)=-\frac{1}{2}(K(\eta) \eta)^{2}+\frac{\left(\eta_{x}-\eta_{x} K(\eta) \eta\right)^{2}}{2\left(1+\eta_{x}^{2}\right)}+K(\eta) \eta
\end{aligned}
$$

and

$$
K(\eta) \xi=-\left(G(\eta)^{-1} \xi_{x}\right)_{x}
$$

Note the equivalent definition

$$
K(\eta) \xi=-\left(\left.\varphi\right|_{y=1+\eta}\right)_{x},
$$

where $\varphi$ is the solution of the boundary-value problem

$$
\begin{array}{ll}
\varphi_{x x}+\varphi_{y y}=0, & 0<y<1+\eta, \\
\varphi_{y}=0, & y=0, \\
\varphi_{y}-\eta_{x} \varphi_{x}=\xi_{x}, & y=1+\eta
\end{array}
$$

(which is unique up to an additive constant).

We proceed by defining the Fourier transform $\hat{u}=\mathcal{F}[u]$ of a function $u$ of a real variable by the formula

$$
\hat{u}(k)=\frac{1}{\sqrt{2 \pi}} \int_{\mathbb{R}} u(x) \mathrm{e}^{-\mathrm{i} k x} \mathrm{~d} x
$$

and using the notation $m(D)$ with $D=-\mathrm{i} \partial_{x}$ for the Fourier multiplier-operator with symbol $m$, so that $m(D) u=\mathcal{F}^{-1}[m \hat{u}]$. The Ansätze (1.5) and (1.9) suggest that the Fourier transform of a solitary wave is concentrated near the points $k= \pm \omega$ (which coincide at $k=0$ when $\left.\beta>\frac{1}{3}\right)$. Indeed, writing $c^{2}=c_{0}^{2}\left(1-\varepsilon^{2}\right)$, one finds that the linearisation of (1.13) at $\varepsilon=0$ is

$$
g(D) \eta=0
$$

where

$$
g(k)=1+\beta k^{2}-c_{0}^{2} k \operatorname{coth} k \geq 0, \quad k \in \mathbb{R},
$$

with equality precisely when $k= \pm \omega$ (so that $g(\omega)=g^{\prime}(\omega)=0$ and $g^{\prime \prime}(\omega)>0$ ). We therefore decompose $\eta$ into the sum of functions $\eta_{1}$ and $\eta_{2}$ whose Fourier transforms $\hat{\eta}_{1}$ and $\hat{\eta}_{2}$ are supported in the region $S=(-\omega-\delta,-\omega+\delta) \cup(\omega-\delta, \omega+\delta)$ (with 
0

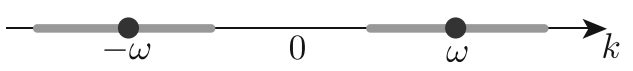

Fig. 3 (a) The support of $\hat{\eta}_{1}$ is contained in the set $S$, where $S=(-\delta, \delta)$ for $\beta>\frac{1}{3}$ (left) and $S=(-\omega-\delta,-\omega+\delta) \cup(\omega-\delta, \omega+\delta)$ for $\beta<\frac{1}{3}$ (right)

$\left.\delta \in\left(0, \frac{1}{3}\right)\right)$ and its complement (see Fig. 3), so that $\eta_{1}=\chi(D) \eta, \eta_{2}=(1-\chi(D)) \eta$, where $\chi$ is the characteristic function of the set $S$. Decomposing (1.13) into

$$
\begin{aligned}
\chi(D)\left(\mathcal{K}\left(\eta_{1}+\eta_{2}\right)-c_{0}^{2}\left(1-\varepsilon^{2}\right) \mathcal{L}\left(\eta_{1}+\eta_{2}\right)\right) & =0, \\
(1-\chi(D))\left(\mathcal{K}\left(\eta_{1}+\eta_{2}\right)-c_{0}^{2}\left(1-\varepsilon^{2}\right) \mathcal{L}\left(\eta_{1}+\eta_{2}\right)\right) & =0,
\end{aligned}
$$

one finds that the second equation can be solved for $\eta_{2}$ as a function of $\eta_{1}$ for sufficiently small values of $\varepsilon>0$; substituting $\eta_{2}=\eta_{2}\left(\eta_{1}\right)$ into the first yields the reduced equation

$$
\chi(D)\left(\mathcal{K}\left(\eta_{1}+\eta_{2}\left(\eta_{1}\right)\right)-c_{0}^{2}\left(1-\varepsilon^{2}\right) \mathcal{L}\left(\eta_{1}+\eta_{2}\left(\eta_{1}\right)\right)=0\right.
$$

for $\eta_{1}$ (see Sect. 3).

Finally, the scaling

$$
\eta_{1}(x)=\varepsilon^{2} \rho(X), \quad X=\varepsilon x,
$$

transforms the reduced equation into

$$
\varepsilon^{-2} g(\varepsilon D) \rho+f(\varepsilon D) \rho+\frac{3}{2} \chi_{0}(\varepsilon D) \rho^{2}+O\left(\varepsilon^{1 / 2}\right)=0
$$

for $\beta>\frac{1}{3}$, while the scaling

$$
\eta_{1}(x)=\frac{1}{2} \varepsilon \zeta(X) \mathrm{e}^{\mathrm{i} \omega x}+\frac{1}{2} \varepsilon \overline{\zeta(X)} \mathrm{e}^{-\mathrm{i} \omega x}, \quad X=\varepsilon x,
$$

transforms the reduced equation into

$$
\varepsilon^{-2} g(\omega+\varepsilon D) \zeta+c_{0}^{2} f(\omega+\varepsilon D) \zeta-a_{3} \chi_{0}(\varepsilon D)\left(|\zeta|^{2} \zeta\right)+O\left(\varepsilon^{1 / 2}\right)=0
$$

for $\beta<\frac{1}{3}$; here $\chi_{0}$ is the characteristic function of the set $(-\delta, \delta)$, the symbol $D$ now means $-\mathrm{i} \partial_{X}$ and precise estimates for the remainder terms are given in Sect. 4. Eqs. (1.21) and (1.23) are termed full dispersion versions of (perturbed) stationary Korteweg-de Vries and nonlinear Schrödinger equations since they retain the linear part of the original equation (1.13); the fully reduced model equations (1.6) and (1.9) are recovered from them in the formal limit $\varepsilon \rightarrow 0$.

Variational versions of this reduction procedure have previously been given by Groves and Wahlén [12]. Starting with the observation that (1.13) is the Euler- 
Lagrange equation for the functional

$$
\mathcal{J}(\eta)=\int_{\mathbb{R}^{2}}\left(\frac{1}{2} \eta^{2}+\beta\left(\sqrt{1+\eta_{x}^{2}+\eta_{z}^{2}}-1\right)-\frac{1}{2} c^{2} \eta K(\eta) \eta\right) \mathrm{d} x \mathrm{~d} z
$$

they use the decomposition $\eta=\eta_{1}+\eta_{2}\left(\eta_{1}\right)$ and scaling of $\eta_{1}$ described above to derive reduced variational functionals for $\rho$ and $\zeta$ whose Euler-Lagrange equations are given to leading order by (1.6) and (1.9). Critical points of the reduced functionals (and hence solitary-wave solutions of the reduced equations) are found by the direct methods of the calculus of variations. In the present paper we apply a more direct perturbative approach introduced by Stefanov and Wright [21] for another full dispersion Kortewegde Vries equation, namely, the Whitham equation (see Ehrnström et al. [9] for a variational treatment of this equation).

The travelling-wave Whitham equation is

$$
c u-m(D) u-u^{2}=0, \quad m(k)=\sqrt{\frac{\tanh k}{k}} .
$$

Noting that its linear dispersion relation has a unique global maximum at $k=0$ (with $c(0)=1)$, one writes $c=1+\varepsilon^{2}$ and seeks solitary waves of the form

$$
u=\varepsilon^{2} w(X), \quad X=\varepsilon x,
$$

so that

$$
\varepsilon^{-2}\left(1+\varepsilon^{2}-m(\varepsilon D)\right) w-w^{2}=0
$$

which can be rewritten as a fixed-point equation of the form

$$
w=\left(1-\frac{1}{6} \partial_{X}^{2}+O\left(\varepsilon^{2}\right)\right)^{-1} w^{2} .
$$

In the formal limit $\varepsilon \rightarrow 0$ we recover the stationary Korteweg-de Vries equation

$$
w-\frac{1}{6} w_{X X}-w^{2}=0
$$

its (unique, symmetric) solitary-wave solution $w^{\star}$ is nondegenerate in the sense that the only bounded solution of its linearisation at $w^{\star}$ is $w_{X}^{\star}$. Restricting to spaces of symmetric functions eliminates this antisymmetric solution of the linearised equation and a solution to (1.24) can be constructed as a perturbation of $w^{\star}$ using the implicitfunction theorem.

In Sect. 5 we apply the above argument to (1.21) and (1.23), first reformulating them as fixed-point equations. The functions $\rho^{\star}$ and $\pm \zeta^{\star}$ are nondegenerate solutions of (1.6) and (1.9) in the sense that the only bounded solutions of their linearisations at $\rho^{\star}$ and $\pm \zeta^{\star}$ are respectively $\rho_{X}^{\star}$ and $\pm \zeta_{X}^{\star}, \pm \mathrm{i} \zeta^{\star}$. Observe that equation (1.13) is invariant under the reflection $\eta(x) \mapsto \eta(-x)$, and the reduction procedure preserves this property: the reduced equation for $\eta_{1}$ is invariant under the reflection 
$\eta_{1}(x) \mapsto \eta_{1}(-x)$, so that (1.21) and (1.23) are invariant under respectively $\rho(x) \mapsto \rho(-x)$ and $\zeta(x) \mapsto \overline{\zeta(-x)}$. Restricting to spaces of symmetric functions thus eliminates the antisymmetric solutions $\rho^{\star \prime}$ and $\pm \zeta_{X}^{\star}, \pm \mathrm{i} \zeta^{\star}$ of the linearised equations, and solutions to (1.21) and (1.23) can be constructed as perturbations of $\rho^{\star}$ and $\pm \zeta^{\star}$ using an appropriate version of the implicit-function theorem.

\subsection{Function Spaces}

We study the equation

$$
\mathcal{K}(\eta)-c_{0}^{2}\left(1-\varepsilon^{2}\right) \mathcal{L}(\eta)=0
$$

in the basic space $\mathcal{X}=H^{2}(\mathbb{R})$, where

$$
H^{s}(\mathbb{R})=\left\{\eta \in \mathcal{S}^{\prime}(\mathbb{R}):\|\eta\|_{s}:=\left\|\left(1+D^{2}\right)^{s / 2} \eta\right\|_{L^{2}(\mathbb{R})}<\infty\right\}, \quad s \geq 0,
$$

are the usual Bessel-potential spaces. The decomposition $\eta=\eta_{1}+\eta_{2}$, where $\eta_{1}=\chi(D) \eta, \eta_{2}=(1-\chi(D)) \eta$, is accommodated by writing $\mathcal{X}$ as the direct sum of $\mathcal{X}_{1}=\chi(D) \mathcal{X}$ and $\mathcal{X}_{2}=(1-\chi(D)) \mathcal{X}$, where $\mathcal{X}_{1}$ and $\mathcal{X}_{2}$ are equipped with respectively the scaled norm

$$
\left\|\eta_{1}\right\| \mid=\left(\int_{\mathbb{R}}\left(1+\varepsilon^{-2}(|k|-\omega)^{2}\right)\left|\hat{\eta}_{1}(k)\right|^{2} \mathrm{~d} k\right)^{1 / 2}
$$

and the usual norm for $H^{2}(\mathbb{R})$. The norm for $\mathcal{X}_{1}$ is so chosen because the final scalings (1.20) and (1.22) transform $\left\|\eta_{1}\right\| \mid$ into a multiple of the standard norm for $H^{1}(\mathbb{R})$, namely, $\left\|\eta_{1}\right\|=\varepsilon^{3 / 2}\|\rho\|_{1}$ and $\left\|\left|\eta_{1}\left\|\mid=\varepsilon^{1 / 2}\right\| \zeta \|_{1}\right.\right.$, and the reduced equations (1.21) and (1.23) are discussed in this space.

The following proposition yields in particular the estimate

$$
\left\|\eta_{1}\right\|_{\infty} \lesssim \varepsilon^{1 / 2}\left\|\eta_{1}\right\|
$$

for the supremum norm of $\eta_{1} \in \mathcal{X}_{1}$. We can also estimate higher-order derivatives of $\eta_{1} \in \mathcal{X}_{1}$ using the fact that the support of $\hat{\eta}_{1}$ is contained in the fixed bounded set $S$, so that, for example

$$
\left\|\eta_{1}\right\|_{n} \lesssim\left\|\eta_{1}\right\|_{0}, \quad\left\|\eta_{1}\right\|_{n, \infty} \lesssim \varepsilon^{1 / 2}\left\|\eta_{1}\right\|
$$

for each $n \in \mathbb{N}_{0}$.

Proposition 1.2 The estimate

$$
\left\|\hat{\eta}_{1}\right\|_{L^{1}(\mathbb{R})} \lesssim \varepsilon^{1 / 2}\|\| \eta_{1} \|
$$

holds for each $\eta_{1} \in \mathcal{X}_{1}$. 
Proof This estimate follows from the calculation

$$
\begin{aligned}
\int_{\mathbb{R}}\left|\hat{\eta}_{1}(k)\right| \mathrm{d} k & =\int_{\mathbb{R}} \frac{\left(1+\varepsilon^{-2}(|k|-\omega)^{2}\right)^{1 / 2}}{\left(1+\varepsilon^{-2}(|k|-\omega)^{2}\right)^{1 / 2}}\left|\hat{\eta}_{1}(k)\right| \mathrm{d} k \\
& \lesssim\|\eta\| \mid\left(\int_{\omega-\delta}^{\omega+\delta} \frac{1}{1+\varepsilon^{-2}(k-\omega)^{2}} \mathrm{~d} k\right)^{1 / 2} \\
& =\frac{1}{2} \pi^{1 / 2} \varepsilon^{1 / 2}\|\eta\| .
\end{aligned}
$$

It is also helpful to use the larger space

$$
\mathcal{Z}=\left\{\eta \in \mathcal{S}^{\prime}(\mathbb{R}):\|\eta\|_{\mathcal{Z}}:=\left\|\hat{\eta}_{1}\right\|_{L^{1}(\mathbb{R})}+\left\|\eta_{2}\right\|_{2}<\infty\right\}
$$

into which $H^{2}(\mathbb{R})$ is continuously embedded. In Sect. 2 we demonstrate that $K(\cdot): \mathcal{Z} \rightarrow \mathcal{L}\left(H^{3 / 2}(\mathbb{R}), H^{1 / 2}(\mathbb{R})\right)$ is analytic at the origin and deduce that $\mathcal{K}, \mathcal{L}$ map the open neighbourhood

$$
U=\left\{\eta \in H^{2}(\mathbb{R}):\|\eta\|_{\mathcal{Z}}<M\right\}
$$

of the origin in $H^{2}(\mathbb{R})$ analytically into $L^{2}(\mathbb{R})$ for sufficiently small values of $M$. Moreover, we take advantage of the estimate

$$
\|\eta\|_{\mathcal{Z}} \lesssim \varepsilon^{1 / 2}\left\|\eta_{1}\right\|+\left\|\eta_{2}\right\|_{2}
$$

for $\eta \in H^{2}(\mathbb{R})$ to obtain estimates for $\mathcal{K}$ and $\mathcal{L}$ which are necessary for the reduction procedure described above (see Sect. 3). Note, however, that in the entirety of the existence theory we work in the fixed subset $U$ of $H^{2}(\mathbb{R})$ (whose elements are 'wellbehaved' functions).

\section{Analyticity}

In this section, we study the operator $K$ given by (1.16) using basic results from the theory of analytic functions in Banach spaces (see the treatise by Buffoni and Toland [6] for a complete account). In particular, we present an elementary proof that $K(\cdot): \mathcal{Z} \rightarrow \mathcal{L}\left(H^{3 / 2}(\mathbb{R}), H^{1 / 2}(\mathbb{R})\right)$, and hence $\mathcal{K}, \mathcal{L}: U \rightarrow L^{2}(\mathbb{R})$, are analytic at the origin (see Sect. 1.3 above). A more comprehensive treatment of the analyticity of operators of Dirichlet-Neumann and Neumann-Dirichlet type in water-wave problems is given by Lannes [18, Ch. 3 and Appendix A] (see also Nicholls and Reitich [19] and $\mathrm{Hu}$ and Nicholls [14]).

We begin with the boundary-value problem (1.17)-(1.19), which is handled using the change of variable

$$
y^{\prime}=\frac{y}{1+\eta}, \quad u\left(x, y^{\prime}\right)=\varphi(x, y)
$$


to map $\Sigma_{\eta}=\{(x, y): x \in \mathbb{R}, 0<y<1+\eta(x)\}$ to the strip $\Sigma=\mathbb{R} \times(0,1)$. Dropping the primes, one finds that (1.17)-(1.19) are transformed into

$$
\begin{array}{ll}
u_{x x}+u_{y y}=\partial_{x} F_{1}(\eta, u)+\partial_{y} F_{2}(\eta, u), & 0<y<1, \\
u_{y}=0, & y=0, \\
u_{y}=F_{2}(\eta, u)+\xi_{x}, & y=1,
\end{array}
$$

where

$$
F_{1}(\eta, u)=-\eta u_{x}+y \eta_{x} u_{y}, \quad F_{2}(\eta, u)=\frac{\eta u_{y}}{1+\eta}+y \eta_{x} u_{x}-\frac{y^{2}}{1+\eta} \eta_{x}^{2} u_{y},
$$

and

$$
K(\eta) \xi=-\left.u_{x}\right|_{y=1}
$$

We discuss (2.1)-(2.3) using the standard Sobolev spaces $H^{n}(\Sigma), n \in \mathbb{N}$, together with $H_{\star}^{n+1}(\Sigma), n \in \mathbb{N}$, which is defined as the completion of

$$
\mathcal{S}(\Sigma, \mathbb{R})=\left\{u \in C^{\infty}(\bar{\Sigma}):|x|^{m}\left|\partial_{x}^{\alpha_{1}} \partial_{y}^{\alpha_{2}} u\right| \text { is bounded for all } m, \alpha_{1}, \alpha_{2} \in \mathbb{N}_{0}\right\}
$$

with respect to the norm

$$
\|u\|_{n+1, \star}^{2}:=\left\|u_{x}\right\|_{H^{n}(\Sigma)}^{2}+\left\|u_{y}\right\|_{H^{n}(\Sigma)}^{2} .
$$

Proposition 2.1 For each $F_{1}, F_{2} \in H^{n}(\Sigma)$ and $\xi \in H^{n+1 / 2}(\mathbb{R}), n \in \mathbb{N}$, the boundaryvalue problem

$$
\begin{array}{ll}
u_{x x}+u_{y y}=\partial_{x} F_{1}+\partial_{y} F_{2}, & 0<y<1, \\
u_{y}=0, & y=0, \\
u_{y}=F_{2}(\eta, u)+\xi_{x}, & y=1,
\end{array}
$$

admits a unique solution $u=S\left(F_{1}, F_{2}, \xi\right)$ in $H_{\star}^{n+1}(\Sigma)$ given (with a slight abuse of notation, in that derivatives should be taken) by the explicit formula

$$
S\left(F_{1}, F_{2}, \xi\right)=\mathcal{F}^{-1}\left[\int_{0}^{1}\left(\mathrm{i} k G(y, \tilde{y}) \hat{F}_{1}-G_{\tilde{y}}(y, \tilde{y}) \hat{F}_{2}\right) \mathrm{d} \tilde{y}-\mathrm{i} k G(y, 1) \hat{\xi}\right],
$$

in which

$$
G(y, \tilde{y})= \begin{cases}-\frac{\cosh |k| y \cosh |k|(1-\tilde{y})}{|k| \sinh |k|}, & 0 \leq y \leq \tilde{y} \leq 1, \\ -\frac{\cosh |k| \tilde{y} \cosh |k|(1-y)}{|k| \sinh |k|}, & 0 \leq \tilde{y} \leq y \leq 1,\end{cases}
$$


so that

$$
\left\|S\left(F_{1}, F_{2}, \xi\right)\right\|_{n+1, \star} \lesssim\left\|F_{1}\right\|_{H^{n}(\Sigma)}+\left\|F_{2}\right\|_{H^{n}(\Sigma)}+\|\xi\|_{n+1 / 2}
$$

Lemma 2.2 For each $\xi \in H^{3 / 2}(\mathbb{R})$ and each sufficiently small $\eta \in \mathcal{Z}$ the boundaryvalue problem (2.1)-(2.3) admits a unique solution $u \in H_{\star}^{2}(\Sigma)$. Furthermore, the mapping $\mathcal{Z} \rightarrow \mathcal{L}\left(H^{3 / 2}(\mathbb{R}), H_{\star}^{2}(\Sigma)\right)$ given by $\eta \mapsto(\xi \mapsto u)$ is analytic at the origin.

Proof Define

$$
T: H_{\star}^{2}(\Sigma) \times \mathcal{Z} \times H^{3 / 2}(\mathbb{R}) \rightarrow H_{\star}^{2}(\Sigma)
$$

by

$$
T(u, \eta, \xi)=u-S\left(F_{1}(\eta, u), F_{2}(\eta, u), \xi\right),
$$

and note that the solutions of (2.1)-(2.3) are precisely the zeros of $T(\cdot, \eta, \xi)$. Using the estimates

$$
\begin{aligned}
&\|\eta w\|_{H^{1}(\Sigma)} \lesssim\|\eta\|_{1, \infty}\|w\|_{H^{1}(\Sigma)} \\
& \lesssim\left(\left\|\eta_{1}\right\|_{1, \infty}+\left\|\eta_{2}\right\|_{2}\right)\|w\|_{H^{1}(\Sigma)}, \\
&\left\|y \eta_{x} w\right\|_{H^{1}(\Sigma)} \lesssim\left(\left\|\eta_{1 x}\right\|_{1, \infty}\|w\|_{H^{1}(\Sigma)}\right.\left.\quad+\left\|\eta_{2 x} w\right\|_{L^{2}(\Sigma)}+\left\|\eta_{2 x} w_{x}\right\|_{L^{2}(\Sigma)}+\left\|\eta_{2 x x} w\right\|_{L^{2}(\Sigma)}\right) \\
& \lesssim\left(\left\|\eta_{1 x}\right\|_{1, \infty}+\left\|\eta_{2 x}\right\|_{\infty}\right)\|w\|_{H^{1}(\Sigma)}+\left\|\eta_{2 x x}\right\|_{0}\|w\|_{H^{1}(\Sigma)} \\
& \lesssim\left(\left\|\eta_{1 x}\right\|_{1, \infty}+\left\|\eta_{2}\right\|_{2}\right)\|w\|_{H^{1}(\Sigma)}, \\
&\left\|y^{2} \eta^{n} \eta_{x}^{2} w\right\|_{H^{1}(\Sigma)} \lesssim\|\eta\|_{1, \infty}^{n}\left(\left\|\eta_{1 x}\right\|_{1, \infty}^{2}\|w\|_{H^{1}(\Sigma)}\right.\left.\quad+\left\|\eta_{2 x}^{2} w\right\|_{L^{2}(\Sigma)}+\left\|\eta_{2 x}^{2} w_{x}\right\|_{L^{2}(\Sigma)}+\left\|\eta_{2 x} \eta_{2 x x} w\right\|_{L^{2}(\Sigma)}\right) \\
& \lesssim \quad\|\eta\|_{1, \infty}^{n}\left(\left(\left\|\eta_{1 x}\right\|_{1, \infty}+\left\|\eta_{2 x}\right\|_{\infty}\right)^{2}\|w\|_{H^{1}(\Sigma)}\right. \\
&\left.+\left\|\eta_{2 x}\right\|_{\infty}\left\|\eta_{2 x x}\right\|_{0}\|w\|_{H^{1}(\Sigma)}\right) \\
& \lesssim\left(\left\|\eta_{1}\right\|_{2, \infty}+\left\|\eta_{2}\right\|_{2}\right)^{n+2}\|w\|_{H^{1}(\Sigma)}
\end{aligned}
$$

(uniformly in $n$ ) and

$$
\left\|\eta_{1}\right\|_{2, \infty}+\left\|\eta_{2}\right\|_{2} \lesssim\left\|\hat{\eta}_{1}\right\|_{L^{1}(\mathbb{R})}+\left\|\eta_{2}\right\|_{2}=\|\eta\|_{\mathcal{Z}}
$$

one finds that the mappings $\mathcal{Z} \times H_{\star}^{2}(\Sigma) \rightarrow H^{1}(\Sigma)$ given by $(\eta, u) \mapsto F_{1}(\eta, u)$ and $(\eta, u) \mapsto F_{2}(\eta, u)$ are analytic at the origin; it follows that $T$ is also analytic at the origin. Furthermore $T(0,0,0)=0$ and

$$
\mathrm{d}_{1} T[0,0,0](u)=u-S\left(F_{1}(0, u), F_{2}(0, u), 0\right)=u-S(0,0,0)=u
$$

(because $S$ is linear and $F_{1}, F_{2}$ are linear in their second arguments), so that $\mathrm{d}_{1} T[0,0,0]=I$ is an isomorphism. By the analytic implicit-function theorem there 
exist open neighbourhoods $N_{1}$ and $N_{2}$ of the origin in $\mathcal{Z}$ and $H^{3 / 2}(\mathbb{R})$ and an analytic function $v: N_{1} \times N_{2} \rightarrow H_{\star}^{2}(\Sigma)$, such that

$$
T(v(\eta, \xi), \eta, \xi)=0
$$

Since $v$ is linear in $\xi$ one can take $N_{2}$ to be the whole space $H^{3 / 2}(\mathbb{R})$.

Corollary 2.3 The mapping $K(\cdot): \mathcal{Z} \rightarrow \mathcal{L}\left(H^{3 / 2}(\mathbb{R}), H^{1 / 2}(\mathbb{R})\right)$ is analytic at the origin.

Corollary 2.4 The formulae (1.14), (1.15) define functions $U \rightarrow L^{2}(\mathbb{R})$ which are analytic at the origin and satisfy $\mathcal{K}(0)=\mathcal{L}(0)=0$.

Proof This result follows from Corollary 2.3 and the facts that $H^{1}(\mathbb{R})$ is a Banach algebra and $\left(u_{1}, u_{2}\right) \mapsto u_{1} u_{2}$ is a bounded bilinear mapping $H^{1 / 2}(\mathbb{R}) \times H^{1 / 2}(\mathbb{R}) \rightarrow$ $L^{2}(\mathbb{R})$ (see Hörmander [13, Theorem 8.3.1]).

In keeping with Lemma 2.2 and Corollaries 2.3 and 2.4 we write

$$
u(\eta, \xi)=\sum_{j=0}^{\infty} u_{j}(\eta, \xi)
$$

where $u_{j}$ is homogeneous of degree $j$ in $\eta$ and linear in $\xi$, and

$$
K(\eta)=\sum_{j=0}^{\infty} K_{j}(\eta), \quad \mathcal{K}(\eta)=\sum_{j=1}^{\infty} \mathcal{K}_{j}(\eta), \quad \mathcal{L}(\eta)=\sum_{j=1}^{\infty} \mathcal{L}_{j}(\eta)
$$

where $K_{j}(\eta), \mathcal{K}_{j}(\eta)$ and $\mathcal{L}_{j}(\eta)$ are homogeneous of degree $j$ in $\eta$ (and we accordingly abbreviate $K_{0}(\eta)$ to $\left.K_{0}\right)$.

Remark 2.5 Note that $K_{j}(\eta)=m_{j}\left(\{\eta\}^{(n)}\right)$, where $m_{j}$ is a bounded, symmetric, $j$-linear mapping $\mathcal{Z}^{j} \rightarrow \mathcal{L}\left(H^{3 / 2}(\mathbb{R}), H^{1 / 2}(\mathbb{R})\right)$.

We examine the first few terms

$$
\begin{aligned}
& \mathcal{K}_{1}(\eta)=\eta-\beta \eta_{x x}, \\
& \mathcal{K}_{2}(\eta)=0 \\
& \mathcal{K}_{3}(\eta)=\frac{3}{2} \beta \eta_{x}^{2} \eta_{x x}
\end{aligned}
$$

and

$$
\begin{aligned}
& \mathcal{L}_{1}(\eta)=K_{0} \eta \\
& \mathcal{L}_{2}(\eta)=\frac{1}{2} \eta_{x}^{2}-\frac{1}{2}\left(K_{0} \eta\right)^{2}+K_{1}(\eta) \eta, \\
& \mathcal{L}_{3}(\eta)=-K_{0} \eta K_{1}(\eta) \eta-\eta_{x}^{2} K_{0} \eta+K_{2}(\eta) \eta
\end{aligned}
$$


in the Maclaurin expansions of $\mathcal{K}$ and $\mathcal{L}$ in more detail since they play a prominent role in our subsequent calculations. We begin by computing explicit expressions for $K_{0}, K_{1}$ and $K_{2}$.

\section{Lemma 2.6}

(i) The operators $K_{0}$ and $K_{1}$ are given by the formulae

$$
K_{0} \xi=f(D) \xi, \quad K_{1}(\eta) \xi=-\left(\eta \xi_{x}\right)_{x}-K_{0}\left(\eta K_{0} \xi\right)
$$

for each $\eta \in H^{2}(\mathbb{R})$ and $\xi \in H^{3 / 2}(\mathbb{R})$.

(ii) The operator $K_{2}$ is given by the formula

$$
K_{2}(\eta) \xi=\frac{1}{2}\left(\eta^{2} K_{0} \xi\right)_{x x}+\frac{1}{2} K_{0}\left(\eta^{2} \xi_{x x}\right)+K_{0}\left(\eta K_{0}\left(\eta K_{0} \xi\right)\right)
$$

under the additional regularity hypothesis that $\eta \in H^{3}(\mathbb{R})$ and $\xi \in H^{5 / 2}(\mathbb{R})$.

Proof (i) The solution to the boundary-value problem

$$
\begin{array}{ll}
u_{0 x x}+u_{0 y y}=0, & 0<y<1, \\
u_{0 y}=0, & y=0, \\
u_{0 y}=\xi_{x}, & y=1,
\end{array}
$$

is

$$
u_{0}=\mathcal{F}^{-1}\left[\frac{\mathrm{i} k}{|k|} \frac{\cosh |k| y}{\sinh |k|} \hat{\xi}\right]
$$

while the solution to the boundary-value problem

$$
\begin{array}{ll}
u_{1 x x}+u_{1 y y}=\left(-\eta u_{0 x}+y \eta_{x} u_{0 y}\right)_{x}+\left(\eta u_{0 y}+y \eta_{x} u_{0 x}\right)_{y}, & 0<y<1, \\
u_{1 y}=0, & y=0, \\
u_{1 y}=\eta u_{0 y}+\eta_{x} u_{0 x}, & y=1,
\end{array}
$$

is

$$
u_{1}=y \eta u_{0 y}+\mathcal{F}^{-1}\left[\frac{\mathrm{i} k}{|k|} \frac{\cosh |k| y}{\sinh |k|} \mathcal{F}\left[\left.\eta u_{0 x}\right|_{y=1}\right]\right]
$$

whence

$$
K_{0} \xi=-\left.u_{0 x}\right|_{y=0}=f(D) \xi, \quad K_{1}(\eta) \xi=-\left.u_{1 x}\right|_{y=0}=-\left(\eta \xi_{x}\right)_{x}-K_{0}\left(\eta K_{0} \xi\right)
$$


(ii) Supposing that $\xi \in H^{5 / 2}(\mathbb{R})$, so that $u^{0} \in H_{\star}^{3}(\Sigma)$, and $\eta \in H^{3}(\mathbb{R})$, so that $u^{1} \in H_{\star}^{3}(\Sigma)$ (see Proposition 2.1), we find that the solution $u^{2} \in H_{\star}^{4}(\Sigma)$ to the boundary-value problem

$$
\begin{aligned}
& u_{2 x x}+u_{2 y y}=\left(-\eta u_{1 x}+y \eta_{x} u_{1 y}\right)_{x} \\
& +\left(\eta u_{1 y}+y \eta_{x} u_{1 x}\right)_{y}-\eta^{2} u_{0 y y}-\left(y^{2} \eta_{x}^{2} u_{0 y}\right)_{y}, \quad 0<y<1, \\
& u_{2 y}=0, \quad y=0, \\
& u_{2 y}=\eta u_{1 y}+\eta_{x} u_{1 x}-\eta^{2} u_{0 y}-\eta_{x}^{2} u_{0 y}, \quad y=1 \text {, }
\end{aligned}
$$

is

$$
\begin{aligned}
u_{2}=- & \frac{1}{2} \eta^{2}\left(y^{2} u_{0 y}\right)_{y}+\eta u_{1 y}-\mathcal{F}^{-1}\left[\frac{\mathrm{i} k}{|k|} \frac{\cosh |k| y}{\sinh |k|} \mathcal{F}\left[\left.\frac{1}{2}\left(\eta^{2} u_{0 y}\right)_{x}\right|_{y=1}\right]\right] \\
& +\mathcal{F}^{-1}\left[\frac{\mathrm{i} k}{|k|} \frac{\cosh |k| y}{\sinh |k|} \mathcal{F}\left[\left.\eta u_{1 x}\right|_{y=1}\right]\right] .
\end{aligned}
$$

It follows that

$$
\begin{aligned}
K_{2}(\eta) \xi= & -\left.u_{2 x}\right|_{y=0} \\
= & -\frac{1}{2}\left(\eta^{2} u_{0 x x}\right)_{x}+\left(\eta^{2} u_{0 y}\right)_{x} \\
& \quad-\left(\eta\left(\eta u_{0 y}+\eta_{x} u_{0 x}\right)\right)_{x}-\frac{1}{2} K_{0}\left(\left(\eta^{2} \xi_{x}\right)_{x}\right)-K_{0}\left(\eta K_{1}(\eta) \xi\right) \\
= & \frac{1}{2}\left(\eta^{2} K_{0} \xi_{x}\right)_{x}+\left(\eta \eta_{x} K_{0} \xi\right)_{x} \\
& \quad-\frac{1}{2} K_{0}\left(\left(\eta^{2} \xi_{x}\right)_{x}\right)+K_{0}\left(\eta\left(\eta \xi_{x}\right)_{x}\right)+K_{0}\left(\eta K_{0}\left(\eta K_{0} \xi\right)\right) \\
= & \frac{1}{2}\left(\eta^{2} K_{0} \xi\right)_{x x}+\frac{1}{2} K_{0}\left(\eta^{2} \xi_{x x}\right)+K_{0}\left(\eta K_{0}\left(\eta K_{0} \xi\right)\right)
\end{aligned}
$$

Remark 2.7 Explicit expressions for $K_{3}, K_{4}, \ldots$ can be computed in a similar fashion. However, computing an expansion in terms of Fourier-multiplier operators in this fashion leads a loss of one derivative at each order. It is therefore necessary to compensate by increasing the regularity of $\xi$ and $\eta$ by one derivative at each order.

\section{Corollary 2.8}

(i) The function $\mathcal{L}_{2}$ is given by the formula

$$
\mathcal{L}_{2}(\eta)=\frac{1}{2}\left(\eta_{x}^{2}-\left(K_{0} \eta\right)^{2}-\left(\eta^{2}\right)_{x x}-2 K_{0}\left(\eta K_{0} \eta\right)\right)
$$

for each $\eta \in H^{2}(\mathbb{R})$.

(ii) The function $\mathcal{L}_{3}$ is given by the formula

$$
\begin{gathered}
\mathcal{L}_{3}(\eta)=K_{0} \eta K_{0}\left(\eta K_{0} \eta\right)+K_{0}\left(\eta K_{0}\left(\eta K_{0} \eta\right)\right)+\eta\left(K_{0} \eta\right) \eta_{x x} \\
+\frac{1}{2} K_{0}\left(\eta^{2} \eta_{x x}\right)+\frac{1}{2}\left(\eta^{2} K_{0} \eta\right)_{x x}
\end{gathered}
$$

under the additional regularity hypothesis that $\eta \in H^{3}(\mathbb{R})$. 
Finally, we record the representation $\mathcal{L}_{2}(\eta)=m(\eta, \eta)$, where

$$
m(u, v)=\frac{1}{2}\left(u_{x} v_{x}-\left(K_{0} u\right)\left(K_{0} v\right)-(u v)_{x x}-K_{0}\left(u K_{0} v+v K_{0} u\right)\right),
$$

which is helpful when performing calculations, and some straightforward estimates for the higher-order parts of $\mathcal{K}$ and $\mathcal{L}$.

Proposition 2.9 The estimate $\|m(u, v)\|_{0} \lesssim\|u\|_{\mathcal{Z}}\|v\|_{2}$ holds for each $u, v \in H^{2}(\mathbb{R})$.

\section{Proposition 2.10}

(i) The quantities

$$
\mathcal{K}_{\mathrm{c}}(\eta):=\sum_{j=3}^{\infty} \mathcal{K}_{j}(\eta), \quad \mathcal{L}_{\mathrm{c}}(\eta):=\sum_{j=3}^{\infty} \mathcal{L}_{j}(\eta)
$$

satisfy the estimates

$$
\begin{aligned}
\left\|\mathcal{K}_{\mathrm{c}}(\eta)\right\|_{0} & \lesssim\|\eta\|_{\mathcal{Z}}^{2}\|\eta\|_{2}, \quad\left\|\mathrm{~d} \mathcal{K}_{\mathrm{c}}[\eta](v)\right\|_{0} \lesssim\|\eta\|_{\mathcal{Z}}^{2}\|v\|_{2}+\|\eta\|_{\mathcal{Z}}\|\eta\|_{2}\|v\|_{\mathcal{Z}}, \\
\left\|\mathcal{L}_{\mathrm{c}}(\eta)\right\|_{0} & \lesssim\|\eta\|_{\mathcal{Z}}^{2}\|\eta\|_{2}, \quad\left\|\mathrm{~d} \mathcal{L}_{\mathrm{c}}[\eta](v)\right\|_{0} \lesssim\|\eta\|_{\mathcal{Z}}^{2}\|v\|_{2}+\|\eta\|_{\mathcal{Z}}\|\eta\|_{2}\|v\|_{\mathcal{Z}}
\end{aligned}
$$

for each $\eta \in U$ and $v \in H^{2}(\mathbb{R})$.

(ii) The quantities

$$
\mathcal{K}_{\mathrm{r}}(\eta):=\sum_{j=4}^{\infty} \mathcal{K}_{j}(\eta), \quad \mathcal{L}_{\mathrm{r}}(\eta):=\sum_{j=4}^{\infty} \mathcal{L}_{j}(\eta)+\frac{1}{2}\left(K_{1}(\eta) \eta\right)^{2}
$$

satisfy the estimates

$$
\begin{aligned}
\left\|\mathcal{K}_{\mathrm{r}}(\eta)\right\|_{0} & \lesssim\|\eta\|_{\mathcal{Z}}^{4}\|\eta\|_{2}, \quad\left\|\mathrm{~d} \mathcal{K}_{\mathrm{r}}[\eta](v)\right\|_{0} \lesssim\|\eta\|_{\mathcal{Z}}^{4}\|v\|_{2}+\|\eta\|_{\mathcal{Z}}^{3}\|\eta\|_{2}\|v\|_{\mathcal{Z}}, \\
\left\|\mathcal{L}_{\mathrm{r}}(\eta)\right\|_{0} & \lesssim\|\eta\|_{\mathcal{Z}}^{3}\|\eta\|_{2}, \quad\left\|\mathrm{~d} \mathcal{L}_{\mathrm{r}}[\eta](v)\right\|_{0} \lesssim\|\eta\|_{\mathcal{Z}}^{3}\|v\|_{2}+\|\eta\|_{\mathcal{Z}}^{2}\|\eta\|_{2}\|v\|_{\mathcal{Z}}
\end{aligned}
$$

for each $\eta \in U$ and $v \in H^{2}(\mathbb{R})$.

Proof These estimates follow from the explicit formulae (2.5), (2.6), together with the calculations

$$
\mathcal{K}(\eta)-\mathcal{K}_{1}(\eta)-\mathcal{K}_{2}(\eta)-\mathcal{K}_{3}(\eta)=\beta\left(1-\frac{3}{2} \eta_{x}^{2}-\frac{1}{\left(1+\eta_{x}^{2}\right)^{3 / 2}}\right) \eta_{x x}
$$

and 


$$
\begin{aligned}
\mathcal{L}(\eta)- & \mathcal{L}_{1}(\eta)-\mathcal{L}_{2}(\eta)-\mathcal{L}_{3}(\eta) \\
=- & \frac{1}{2}\left(K_{1}(\eta) \eta\right)^{2}-\frac{1}{2}\left(K_{\mathrm{r}}(\eta) \eta\right)^{2}-\left(K_{0} \eta+K_{1}(\eta)\right) K_{\mathrm{r}}(\eta) \eta-\eta_{x}^{2}\left(K_{1}(\eta) \eta+K_{\mathrm{r}}(\eta) \eta\right) \\
& \quad+\frac{1}{2} \eta_{x}^{2}(K(\eta) \eta)^{2}-\frac{\eta_{x}^{2}}{2\left(1+\eta_{x}^{2}\right)}\left(\eta_{x}-\eta_{x} K(\eta) \eta\right)^{2}+\left(K_{\mathrm{r}}(\eta)-K_{2}(\eta)\right) \eta,
\end{aligned}
$$

where

$$
K_{\mathrm{r}}(\eta)=\sum_{j=2}^{\infty} K_{j}(\eta)
$$

\section{Reduction}

In this section, we reduce the equation

$$
\mathcal{K}(\eta)-c_{0}^{2}\left(1-\varepsilon^{2}\right) \mathcal{L}(\eta)=0
$$

to a locally equivalent equation for $\eta_{1}$. Clearly $\eta \in U$ satisfies (3.1) if and only if

$$
\begin{aligned}
\chi(D)\left(\mathcal{K}\left(\eta_{1}+\eta_{2}\right)-c_{0}^{2}\left(1-\varepsilon^{2}\right) \mathcal{L}\left(\eta_{1}+\eta_{2}\right)\right) & =0, \\
(1-\chi(D))\left(\mathcal{K}\left(\eta_{1}+\eta_{2}\right)-c_{0}^{2}\left(1-\varepsilon^{2}\right) \mathcal{L}\left(\eta_{1}+\eta_{2}\right)\right) & =0,
\end{aligned}
$$

and these equations can be rewritten as

$$
\begin{aligned}
g(D) \eta_{1}+c_{0}^{2} \varepsilon^{2} K_{0} \eta_{1}+\chi(D) \mathcal{N}\left(\eta_{1}+\eta_{2}\right) & =0 \\
g(D) \eta_{2}+c_{0}^{2} \varepsilon^{2} K_{0} \eta_{2}+(1-\chi(D)) \mathcal{N}\left(\eta_{1}+\eta_{2}\right) & =0
\end{aligned}
$$

in which

$$
\mathcal{N}(\eta)=\mathcal{K}_{\mathrm{c}}(\eta)-c_{0}^{2}\left(1-\varepsilon^{2}\right)\left(\mathcal{L}_{2}(\eta)+\mathcal{L}_{\mathrm{c}}(\eta)\right)
$$

We proceed by writing (3.3) as a fixed-point equation for $\eta_{2}$ using Proposition 3.1, which follows from the fact that $g(k) \gtrsim|k|^{2}$ for $k \notin S$, and solving it for $\eta_{2}$ as a function of $\eta_{1}$ using Theorem 3.2, which is proved by a straightforward application of the contraction mapping principle. Substituting $\eta_{2}=\eta_{2}\left(\eta_{1}\right)$ into (3.2) yields a reduced equation for $\eta_{1}$. Note that the reduced equation is invariant under the reflection $\eta_{1}(x) \mapsto \eta_{1}(-x)$, which is inherited from the invariance of (3.1) under the reflection $\eta(x) \mapsto \eta(-x)$ (see below).

Proposition 3.1 The mapping $(1-\chi(D)) g(D)^{-1}$ is a bounded linear operator $L^{2}(\mathbb{R}) \rightarrow \mathcal{X}_{2}$. 
Theorem 3.2 Let $\mathcal{X}_{1}, \mathcal{X}_{2}$ be Banach spaces, $X_{1}, X_{2}$ be closed, convex sets in, respectively, $\mathcal{X}_{1}, \mathcal{X}_{2}$ containing the origin and $\mathcal{G}: X_{1} \times X_{2} \rightarrow \mathcal{X}_{2}$ be a smooth function. Suppose that there exists a continuous function $r: X_{1} \rightarrow[0, \infty)$, such that

$$
\left\|\mathcal{G}\left(x_{1}, 0\right)\right\| \leq \frac{1}{2} r, \quad\left\|\mathrm{~d}_{2} \mathcal{G}\left[x_{1}, x_{2}\right]\right\| \leq \frac{1}{3}
$$

for each $x_{2} \in \bar{B}_{r}(0) \subseteq X_{2}$ and each $x_{1} \in X_{1}$.

Under these hypotheses there exists for each $x_{1} \in X_{1}$ a unique solution $x_{2}=x_{2}\left(x_{1}\right)$ of the fixed-point equation $x_{2}=\mathcal{G}\left(x_{1}, x_{2}\right)$ satisfying $x_{2}\left(x_{1}\right) \in \bar{B}_{r}(0)$. Moreover $x_{2}\left(x_{1}\right)$ is a smooth function of $x_{1} \in X_{1}$ and in particular satisfies the estimate

$$
\left\|\mathrm{d} x_{2}\left[x_{1}\right]\right\| \leq 2\left\|\mathrm{~d}_{1} \mathcal{G}\left[x_{1}, x_{2}\left(x_{1}\right)\right]\right\| .
$$

\subsection{Strong Surface Tension}

Suppose that $\beta>\frac{1}{3}$. We write (3.3) in the form

$$
\eta_{2}=-(1-\chi(D)) g(D)^{-1}\left(\varepsilon^{2} K_{0} \eta_{2}+\mathcal{N}\left(\eta_{1}+\eta_{2}\right)\right)
$$

and apply Theorem 3.2 with

$$
X_{1}=\left\{\eta_{1} \in \mathcal{X}_{1}:\left\|\eta_{1}\right\| \leq R_{1}\right\}, \quad X_{2}=\left\{\eta_{2} \in \mathcal{X}_{2}:\left\|\eta_{2}\right\|_{2} \leq R_{2}\right\}
$$

the function $\mathcal{G}$ is given by the right-hand side of (3.4). Using Proposition 1.2 one can guarantee that $\left\|\hat{\eta}_{1}\right\|_{L^{1}\left(\mathbb{R}^{2}\right)}<\frac{1}{2} M$ for all $\eta_{1} \in X_{1}$ for an arbitrarily large value of $R_{1}$; the value of $R_{2}$ is constrained by the requirement that $\left\|\eta_{2}\right\|_{2}<\frac{1}{2} M$ for all $\eta_{2} \in X_{2}$.

Lemma 3.3 The estimates

(i) $\left\|\mathcal{G}\left(\eta_{1}, \eta_{2}\right)\right\|_{2} \lesssim \varepsilon^{1 / 2}\left\|\eta_{1}\right\|\left\|^{2}+\varepsilon^{1 / 2}\right\|\left\|\eta_{1}\right\|\|\| \eta_{2}\left\|_{2}+\right\|\left\|\eta_{1}\right\|\left\|\eta_{2}\right\|_{2}^{2}+\left\|\eta_{2}\right\|_{2}^{2}+\varepsilon^{2}\left\|\eta_{2}\right\|_{2}$,

(ii) $\left\|\mathrm{d}_{1} \mathcal{G}\left[\eta_{1}, \eta_{2}\right]\right\|_{\mathcal{L}\left(\mathcal{X}_{1}, \mathcal{X}_{2}\right)} \lesssim \varepsilon^{1 / 2}\left\|\eta_{1}\right\|+\varepsilon^{1 / 2}\left\|\eta_{2}\right\|_{2}+\left\|\eta_{2}\right\|_{2}^{2}$,

(iii) $\left\|\mathrm{d}_{2} \mathcal{G}\left[\eta_{1}, \eta_{2}\right]\right\|_{\mathcal{L}\left(\mathcal{X}_{1}, \mathcal{X}_{2}\right)} \lesssim \varepsilon^{1 / 2}\left\|\left|\eta_{1}\right|\right\|+\left\|\eta_{1}\right\|\left\|\eta_{2}\right\|_{2}+\left\|\eta_{2}\right\|_{2}+\varepsilon^{2}$

hold for each $\eta_{1} \in X_{1}$ and $\eta_{2} \in X_{2}$.

Proof Observe that

$$
\left\|K_{0} \eta_{2}\right\|_{0} \lesssim\left\|\eta_{2}\right\|_{2}
$$

and using Propositions 2.9 and 2.10(i), one finds that $\left\|\mathcal{L}_{2}\left(\eta_{1}+\eta_{2}\right)\right\|_{0}=\left\|m\left(\left\{\eta_{1}+\eta_{2}\right\}^{(2)}\right)\right\|_{0} \lesssim\left\|\eta_{1}\right\|_{\mathcal{Z}}\left\|\eta_{1}\right\|_{2}+\left\|\eta_{1}\right\|_{\mathcal{Z}}\left\|\eta_{2}\right\|_{2}+\left\|\eta_{2}\right\|_{\mathcal{Z}}\left\|\eta_{2}\right\|_{2}$ and

$$
\left\|\mathcal{K}_{\mathrm{c}}\left(\eta_{1}+\eta_{2}\right)\right\|_{0},\left\|\mathcal{L}_{\mathrm{c}}\left(\eta_{1}+\eta_{2}\right)\right\|_{0} \lesssim\left\|\eta_{1}+\eta_{2}\right\|_{\mathcal{Z}}^{2}\left\|\eta_{1}+\eta_{2}\right\|_{2}
$$


part (i) follows from these estimates and inequality (1.25). Parts (ii) and (iii) are obtained in a similar fashion.

Theorem 3.4 Equation (3.4) has a unique solution $\eta_{2} \in X_{2}$ which depends smoothly upon $\eta_{1} \in X_{1}$ and satisfies the estimates

$$
\left\|\eta_{2}\left(\eta_{1}\right)\right\|_{2} \lesssim \varepsilon^{1 / 2}\left\|\eta_{1}\right\|^{2}, \quad\left\|\mathrm{~d} \eta_{2}\left[\eta_{1}\right]\right\| \mathcal{L}_{\left(\mathcal{X}_{1}, \mathcal{X}_{2}\right)} \lesssim \varepsilon^{1 / 2}\left\|\eta_{1}\right\|
$$

Proof Choosing $R_{2}$ and $\varepsilon$ sufficiently small and setting $r\left(\eta_{1}\right)=\sigma \varepsilon^{1 / 2}\left\|\eta_{1}\right\|^{2}$ for a sufficiently large value of $\sigma>0$, one finds that

$$
\left\|\mathcal{G}\left(\eta_{1}, 0\right)\right\|_{2} \lesssim \frac{1}{2} r\left(\eta_{1}\right), \quad\left\|\mathrm{d}_{2} \mathcal{G}\left[\eta_{1}, \eta_{3}\right]\right\|_{\mathcal{L}\left(\mathcal{X}_{2}, \mathcal{X}_{2}\right)} \lesssim \varepsilon^{1 / 2}
$$

for $\eta_{1} \in X_{1}$ and $\eta_{2} \in \bar{B}_{r\left(\eta_{1}\right)}(0) \subset X_{2}$ (Lemma 3.3(i), (iii)). Theorem 3.2 asserts that equation (3.4) has a unique solution $\eta_{2}$ in $\bar{B}_{r\left(\eta_{1}\right)}(0) \subset X_{2}$ which depends smoothly upon $\eta_{1} \in X_{1}$, and the estimate for its derivative follows from Lemma 3.3(ii).

Substituting $\eta_{2}=\eta_{2}\left(\eta_{1}\right)$ into (3.2) yields the reduced equation

$$
g(D) \eta_{1}+c_{0}^{2} \varepsilon^{2} K_{0} \eta_{1}+\chi(D) \mathcal{N}\left(\eta_{1}+\eta_{2}\left(\eta_{1}\right)\right)=0
$$

for $\eta_{1} \in X_{1}$. Observe that this equation is invariant under the reflection $\eta_{1}(x) \mapsto \eta_{1}(-x)$; a familiar argument shows that it is inherited from the corresponding invariance of (3.2), (3.4) under $\eta_{1}(x) \mapsto \eta_{1}(-x), \eta_{2}(x) \mapsto \eta_{2}(-x)$ when applying Theorem 3.2.

\subsection{Weak Surface Tension}

Suppose that $\beta<\frac{1}{3}$. Since $\chi(D) \mathcal{L}_{2}\left(\eta_{1}\right)=0$ the nonlinear term in (3.2) is at leading order cubic in $\eta_{1}$, so that this equation may be rewritten as

$$
g(D) \eta_{1}+c_{0}^{2} \varepsilon^{2} K_{0} \eta_{1}+\chi(D)\left(\mathcal{N}\left(\eta_{1}+\eta_{2}\right)+c_{0}^{2}\left(1-\varepsilon^{2}\right) \mathcal{L}_{2}\left(\eta_{1}\right)\right)=0
$$

To compute the reduced equation for $\eta_{1}$ we need an explicit formula for the leadingorder quadratic part of $\eta_{2}\left(\eta_{1}\right)$, which is evidently given by

$$
F\left(\eta_{1}\right):=c_{0}^{2}\left(1-\varepsilon^{2}\right)(1-\chi(D)) g(D)^{-1} \mathcal{L}_{2}\left(\eta_{1}\right) .
$$

It is convenient to write $\eta_{2}=F\left(\eta_{1}\right)+\eta_{3}$ and (3.3) in the form

$$
\begin{aligned}
\eta_{3}=- & (1-\chi(D)) g(D)^{-1} \\
& \left(c_{0}^{2}\left(1-\varepsilon^{2}\right) \mathcal{L}_{2}\left(\eta_{1}\right)+\mathcal{N}\left(\eta_{1}+F\left(\eta_{1}\right)+\eta_{3}\right)+c_{0}^{2} \varepsilon^{2} K_{0}\left(F\left(\eta_{1}\right)+\eta_{3}\right)\right)
\end{aligned}
$$


(with the requirement that $\eta_{1}+F\left(\eta_{1}\right)+\eta_{3} \in U$ ). We apply Theorem 3.2 to equation (3.8) with

$$
X_{1}=\left\{\eta_{1} \in \mathcal{X}_{1}:\left\|\eta_{1}\right\| \leq R_{1}\right\}, \quad X_{3}=\left\{\eta_{3} \in \mathcal{X}_{2}:\left\|\eta_{3}\right\|_{3} \leq R_{3}\right\}
$$

the function $\mathcal{G}$ is given by the right-hand side of (3.8). (Here we write $X_{3}$ rather than $X_{2}$ for notational clarity.) Using Proposition 1.2 one can guarantee that $\left\|\hat{\eta}_{1}\right\|_{L^{1}(\mathbb{R})}<\frac{1}{2} M$ for all $\eta_{1} \in X_{1}$ for an arbitrarily large value of $R_{1}$; the value of $R_{3}$ is constrained by the requirement that $\left\|F\left(\eta_{1}\right)+\eta_{3}\right\|_{2}<\frac{1}{2} M$ for all $\eta_{1} \in X_{1}$ and $\eta_{3} \in X_{3}$, so that $\eta_{1}+F\left(\eta_{1}\right)+\eta_{3} \in U$ (Proposition 3.5 below asserts that $\left\|F\left(\eta_{1}\right)\right\|_{2}=O\left(\varepsilon^{1 / 2}\right.$ ) uniformly over $\left.\eta_{1} \in X_{1}\right)$.

Proposition 3.5 The estimates

$$
\left\|F\left(\eta_{1}\right)\right\|_{2} \lesssim \varepsilon^{1 / 2}\left\|\eta_{1}\right\|^{2}, \quad\left\|\mathrm{~d} F\left[\eta_{1}\right]\right\|_{\mathcal{L}\left(\mathcal{X}_{1}, \mathcal{X}_{2}\right)} \lesssim \varepsilon^{1 / 2}\left\|\eta_{1}\right\|
$$

hold for each $\eta_{1} \in X_{1}$.

Proof This result follows from the formula

$$
F\left(\eta_{1}\right)=c_{0}^{2}\left(1-\varepsilon^{2}\right)(1-\chi(D)) g(D)^{-1} m_{2}\left(\eta_{1}, \eta_{1}\right),
$$

Proposition 2.9 and inequality (1.25).

Remark 3.6 Noting that

$$
K_{0} F\left(\eta_{1}\right)=c_{0}^{2}\left(1-\varepsilon^{2}\right)(1-\chi(D)) g(D)^{-1} f(D) m_{2}\left(\eta_{1}, \eta_{1}\right)
$$

and that $m\left(u_{1}, v_{1}\right)$ has compact support for all $u_{1}, v_{1} \in \mathcal{X}_{1}$, one finds that $K_{0} F\left(\eta_{1}\right)$ satisfies the same estimates as $F\left(\eta_{1}\right)$.

Lemma 3.7 The quantity

$$
\mathcal{N}_{1}\left(\eta_{1}, \eta_{3}\right)=\mathcal{L}_{2}\left(\eta_{1}+F\left(\eta_{1}\right)+\eta_{3}\right)-\mathcal{L}_{2}\left(\eta_{1}\right)
$$

satisfies the estimates

(i) $\left\|\mathcal{N}_{1}\left(\eta_{1}, \eta_{3}\right)\right\|_{0} \lesssim \varepsilon\|\| \eta_{1}\|\|^{3}+\varepsilon^{1 / 2}\left\|\left|\eta_{1}\left\|\left.\right|^{2}\right\| \eta_{3}\left\|_{2}+\varepsilon^{1 / 2}\right\|\right| \eta_{1}\right\|\|\| \eta_{3}\left\|_{2}+\right\| \eta_{3} \|_{2}^{2}$,

(ii) $\left\|\mathrm{d}_{1} \mathcal{N}_{1}\left[\eta_{1}, \eta_{3}\right]\right\|_{\mathcal{L}\left(\mathcal{X}_{1}, L^{2}(\mathbb{R})\right)} \lesssim \varepsilon\|\| \eta_{1}\|\|^{2}+\varepsilon^{1 / 2}\left\|\eta_{1}\right\|\left\|\eta_{3}\right\|_{2}+\varepsilon^{1 / 2}\left\|\eta_{3}\right\|_{2}$,

(iii) $\left\|\mathrm{d}_{2} \mathcal{N}_{1}\left[\eta_{1}, \eta_{3}\right]\right\|_{\mathcal{L}\left(\mathcal{X}_{2}, L^{2}(\mathbb{R})\right)} \lesssim \varepsilon^{1 / 2}\left\|\left|\eta_{1}\|\mid+\| \eta_{3} \|_{2}\right.\right.$

for each $\eta_{1} \in X_{1}$ and $\eta_{3} \in X_{3}$.

Proof We estimate

$$
\mathcal{N}_{1}\left(\eta_{1}, \eta_{3}\right)=2 m\left(\eta_{1}, F\left(\eta_{1}\right)+\eta_{3}\right)+m\left(F\left(\eta_{1}\right)+\eta_{3}, F\left(\eta_{1}\right)+\eta_{3}\right)
$$

and its derivatives, which are computed using the chain rule, using Propositions 2.9 and 3.5 and inequality (1.25). 


\section{Lemma 3.8 The quantity}

$$
\mathcal{N}_{2}\left(\eta_{1}, \eta_{3}\right)=\mathcal{K}_{\mathrm{c}}\left(\eta_{1}+F\left(\eta_{1}\right)+\eta_{3}\right)-c_{0}^{2}\left(1-\varepsilon^{2}\right)\left(\mathcal{L}_{\mathrm{c}}\left(\eta_{1}+F\left(\eta_{1}\right)+\eta_{3}\right)\right.
$$

satisfies the estimates

(i) $\left\|\mathcal{N}_{2}\left(\eta_{1}, \eta_{3}\right)\right\|_{0} \lesssim\left(\varepsilon^{1 / 2}\left\|\eta_{1}\right\| \mid+\left\|\eta_{3}\right\|_{2}\right)^{2}\left(\left\|\eta_{1}\right\| \mid+\left\|\eta_{3}\right\|_{2}\right)$,

(ii) $\left\|\mathrm{d}_{1} \mathcal{N}_{2}\left[\eta_{1}, \eta_{3}\right]\right\|_{\mathcal{L}\left(\mathcal{X}_{1}, L^{2}(\mathbb{R})\right)} \lesssim\left(\varepsilon^{1 / 2}\left\|\eta_{1}\right\| \mid+\left\|\eta_{3}\right\|_{2}\right)^{2}$,

(iii) $\left\|\mathrm{d}_{2} \mathcal{N}_{2}\left[\eta_{1}, \eta_{3}\right]\right\|_{\mathcal{L}\left(\mathcal{X}_{2}, L^{2}(\mathbb{R})\right)} \lesssim\left(\varepsilon^{1 / 2}\left\|\left|\eta_{1}\|\mid+\| \eta_{3} \|_{2}\right)\left(\left\|\eta_{1}\right\| \mid+\left\|\eta_{3}\right\|_{2}\right)\right.\right.$

for each $\eta_{1} \in X_{1}$ and $\eta_{3} \in X_{3}$.

Proof We estimate $\mathcal{N}_{2}$ and its derivatives, which are computed using the chain rule, using Propositions 2.10(i) and 3.5 and inequality (1.25).

Altogether we have established the following estimates for $\mathcal{G}$ and its derivatives (see Remark 3.6 and Lemmata 3.7 and 3.8).

\section{Lemma 3.9 The estimates}

(i) $\left\|\mathcal{G}\left(\eta_{1}, \eta_{3}\right)\right\|_{2} \lesssim\left(\varepsilon^{1 / 2}\left\|\left|\eta_{1}\|\mid+\| \eta_{3} \|_{2}\right)^{2}\left(1+\left\|\left|\eta_{1}\|\mid+\| \eta_{3} \|_{2}\right)+\varepsilon^{2}\right\| \eta_{3} \|_{2}\right.\right.\right.$,

(ii) $\left\|\mathrm{d}_{1} \mathcal{G}\left[\eta_{1}, \eta_{3}\right]\right\|_{\mathcal{L}\left(\mathcal{X}_{1}, \mathcal{X}_{2}\right)} \lesssim\left(\varepsilon^{1 / 2}\|\| \eta_{1}\|\mid+\| \eta_{3} \|_{2}\right)\left(\varepsilon^{1 / 2}+\varepsilon^{1 / 2}\left\|\eta_{1}\right\|\|+\| \eta_{3} \|_{2}\right)$,

(iii) $\left\|\mathrm{d}_{2} \mathcal{G}\left[\eta_{1}, \eta_{3}\right]\right\|_{\mathcal{L}\left(\mathcal{X}_{2}, \mathcal{X}_{2}\right)} \lesssim\left(\varepsilon^{1 / 2}\left|\left\|\eta_{1}\right\|\right|+\left\|\eta_{3}\right\|_{2}\right)\left(1+\left\|\eta_{1}\right\| \mid+\left\|\eta_{3}\right\|_{2}\right)+\varepsilon^{2}$

hold for each $\eta_{1} \in X_{1}$ and $\eta_{3} \in X_{3}$.

Theorem 3.10 Equation (3.8) has a unique solution $\eta_{3} \in X_{3}$ which depends smoothly upon $\eta_{1} \in X_{1}$ and satisfies the estimates

$$
\left\|\eta_{3}\left(\eta_{1}\right)\right\|_{2} \lesssim \varepsilon\|\| \eta_{1}\left\|^{2}, \quad\right\| \mathrm{d} \eta_{3}\left[\eta_{1}\right]\left\|_{\mathcal{L}\left(\mathcal{X}_{1}, \mathcal{X}_{2}\right)} \lesssim \varepsilon\right\|\left\|\eta_{1}\right\| .
$$

Proof Choosing $R_{3}$ and $\varepsilon$ sufficiently small and setting $r\left(\eta_{1}\right)=\sigma \varepsilon\|\| \eta_{1}\|\|^{2}$ for a sufficiently large value of $\sigma>0$, one finds that

$$
\left\|\mathcal{G}\left(\eta_{1}, 0\right)\right\|_{2} \lesssim \frac{1}{2} r\left(\eta_{1}\right), \quad\left\|\mathrm{d}_{2} \mathcal{G}\left[\eta_{1}, \eta_{3}\right]\right\|_{\mathcal{L}\left(\mathcal{X}_{2}, \mathcal{X}_{2}\right)} \lesssim \varepsilon^{1 / 2}
$$

for $\eta_{1} \in X_{1}$ and $\eta_{3} \in \bar{B}_{r\left(\eta_{1}\right)}(0) \subset X_{3}$ (Lemma 3.9(i), (iii)). Theorem 3.2 asserts that equation (3.8) has a unique solution $\eta_{3}$ in $\bar{B}_{r\left(\eta_{1}\right)}(0) \subset X_{3}$ which depends smoothly upon $\eta_{1} \in X_{1}$, and the estimate for its derivative follows from Lemma 3.9(ii).

Substituting $\eta_{2}=F\left(\eta_{1}\right)+\eta_{3}\left(\eta_{1}\right)$ into (3.6) yields the reduced equation

$$
\begin{aligned}
& g(D) \eta_{1}+c_{0}^{2} \varepsilon^{2} K_{0} \eta_{1} \\
& \quad+\chi(D)\left(\mathcal{N}\left(\eta_{1}+F\left(\eta_{1}\right)+\eta_{3}\left(\eta_{2}\right)\right)+c_{0}^{2}\left(1-\varepsilon^{2}\right) \mathcal{L}_{2}\left(\eta_{1}\right)\right)=0
\end{aligned}
$$

for $\eta_{1} \in X_{1}$. This equation is also invariant under the reflection $\eta_{1}(x) \mapsto \eta_{1}(-x)$; it is inherited from the invariance of (3.6), (3.8) under $\eta_{1}(x) \mapsto \eta_{1}(-x), \eta_{3}(x) \mapsto \eta_{3}(-x)$ when applying Theorem 3.2. 


\section{Derivation of the Reduced Equation}

In this section we compute the leading-order terms in the reduced equations (3.5) and (3.11) and hence derive the perturbed full dispersion Korteweg-de Vries and nonlinear Schrödinger equations announced in Sect. 1. The main steps are approximating the Fourier-multiplier operators appearing in lower-order terms by constants, estimating higher-order terms and performing the scalings (1.20) and (1.22).

It is convenient to introduce some additional notation to estimate higher-order 'remainder' terms.

\section{Definition 4.1}

(i) The symbol $\mathcal{O}\left(\varepsilon^{\gamma}\left\|\eta_{1}\right\|^{r}\right)$ denotes a smooth function $\mathcal{R}: X_{1} \rightarrow L^{2}(\mathbb{R})$ which satisfies the estimates

$$
\left\|\mathcal{R}\left(\eta_{1}\right)\right\|_{0} \lesssim \varepsilon^{\gamma}\left\|\eta_{1}\right\|^{r}, \quad\left\|\mathrm{~d} \mathcal{R}\left[\eta_{1}\right]\right\|_{\mathcal{L}\left(\mathcal{X}_{1}, L^{2}(\mathbb{R})\right)} \lesssim \varepsilon^{\gamma}\left\|\eta_{1}\right\|^{r-1}
$$

for each $\eta_{1} \in X_{1}$ (where $\gamma \geq 0, r \geq 1$ ), and the underscored notation $\underline{\mathcal{O}}\left(\left.\varepsilon^{\gamma}\left\|\eta_{1}\right\|\right|^{r}\right)$ indicates additionally that the Fourier transform of $\mathcal{R}\left(\eta_{1}\right)$ lies in a fixed compact set (independently of $\varepsilon$ and uniformly over $\eta_{1} \in X_{1}$ ). Furthermore

$$
\begin{aligned}
& \underline{\mathcal{O}}_{0}\left(\left.\varepsilon^{\gamma}\left\|\eta_{1}\right\|\right|^{r}\right):=\chi_{0}(D) \mathcal{O}\left(\varepsilon^{\gamma}\left\|\left|\eta_{1} \|\right|^{r}\right),\right. \\
& \underline{\mathcal{O}}_{+}\left(\left.\varepsilon^{\gamma}\left\|\eta_{1}\right\|\right|^{r}\right):=\chi^{+}(D) \mathcal{O}\left(\varepsilon^{\gamma}\left\|\left|\eta_{1} \|\right|^{r}\right),\right.
\end{aligned}
$$

where $\chi_{0}$ and $\chi^{+}$are the characteristic functions of the sets $(-\delta, \delta)$ and $(\omega-\delta, \omega+\delta)$ (for $\omega>0)$.

(ii) The symbol $\underline{\mathcal{O}}_{n}^{\varepsilon}\left(\|u\|_{1}^{r}\right)$ denotes $\chi_{0}(\varepsilon D) \mathcal{R}(u)$, where $\mathcal{R}$ is a smooth function $B_{R}(0) \subseteq \chi_{0}(\varepsilon D) H^{1}(\mathbb{R}) \rightarrow H^{n}(\mathbb{R})$ or $B_{R}(0) \subseteq H^{1}(\mathbb{R}) \rightarrow H^{n}(\mathbb{R})$ which satisfies the estimates

$$
\|\mathcal{R}(u)\|_{n} \lesssim\|u\|_{1}^{r}, \quad\|\mathrm{~d} \mathcal{R}[u]\|_{\mathcal{L}\left(H^{1}(\mathbb{R}), H^{n}(\mathbb{R})\right)} \lesssim\|u\|_{1}^{r-1}
$$

for each $u \in B_{R}(0)$ (with $r \geq 1, n \geq 0$ ).

\subsection{Strong Surface Tension}

The leading-order terms in the reduced equation

$$
g(D) \eta_{1}+c_{0}^{2} \varepsilon^{2} K_{0} \eta_{1}+\chi(D) \mathcal{N}\left(\eta_{1}+\eta_{2}\left(\eta_{1}\right)\right)=0
$$

derived in Sect. 3.1 are computed by approximating the operators $\partial_{x}$ and $K_{0}$ in the quadratic part of the equation by constants. 
Proposition 4.2 The estimates

(i) $\eta_{1 x}=\underline{\mathcal{O}}_{0}\left(\varepsilon\left\|\left|\eta_{1}\right|\right\|\right)$,

(ii) $K_{0} \eta_{1}=\eta_{1}+\underline{\mathcal{O}}_{0}\left(\varepsilon\left\|\left|\eta_{1}\right|\right\|\right)$,

(iii) $K_{0} \eta_{1}^{2}=\eta_{1}^{2}+\underline{\mathcal{O}}\left(\varepsilon^{3 / 2}\left\|\mid \eta_{1}\right\|\right)$

hold for each $\eta_{1} \in X_{1}$.

Proof Note that

$$
\left\|\eta_{1 x}\right\|_{0}^{2}=\left\||k| \hat{\eta}_{1}\right\|_{0}^{2} \leq \varepsilon^{2}\left\|\mid \eta_{1}\right\|^{2}
$$

and

$$
\left\|\left(K_{0}-I\right) \eta_{1}\right\|_{0}^{2}=\left\|(|k| \operatorname{coth}|k|-1) \hat{\eta}_{1}\right\|_{0}^{2} \lesssim\left\||k|^{2} \hat{\eta}_{1}\right\|_{0}^{2} \leq \varepsilon^{2}\|\| \eta_{1} \|^{2}
$$

the corresponding estimates for their derivatives are trivially satisfied since the operators are linear. The quantity to be estimated in (iii) is quadratic in $\eta_{1}$; it therefore suffices to estimate the corresponding bilinear operator. The argument used above yields

$$
\begin{aligned}
\left\|\left(K_{0}-I\right)\left(u_{1} v_{1}\right)\right\|_{0} \\
\quad \lesssim\left\|| k | \int _ { \mathbb { R } } \left|\hat{u}_{1}(k-s)\left\|\hat{v}_{1}(s) \mid \mathrm{d} s\right\|_{0}\right.\right. \\
\quad \lesssim\left\|\int_{\mathbb{R}}\left|k-s\left\|\hat{u}_{1}(k-s)\right\| \hat{v}_{1}(s)\right| \mathrm{d} s+\int_{\mathbb{R}}|s|\left|\hat{u}_{1}(k-s)\left\|\hat{v}_{1}(s) \mid \mathrm{d} s\right\|_{0}\right.\right. \\
\quad \lesssim\left\||k| \hat{u}_{1}\right\|_{0}\left\|\hat{v}_{1}\right\|_{L^{1}(\mathbb{R})}+\left\|\hat{u}_{1}\right\|_{L^{1}(\mathbb{R})}\left\||k| \hat{v}_{1}\right\|_{0} \\
\quad \lesssim \varepsilon^{3 / 2}\|\| u_{1}\left\|\left|\left\|v_{1}\right\|\right|\right.
\end{aligned}
$$

for each $u_{1}, v_{1} \in X_{1}$, where we have also used Young's inequality.

\section{Lemma 4.3 The estimate}

$$
\mathcal{L}_{2}\left(\eta_{1}+\eta_{2}\left(\eta_{1}\right)\right)=-\frac{3}{2} \eta_{1}^{2}+\mathcal{O}\left(\varepsilon\left\|\mid \eta_{1}\right\| \|^{2}\right)
$$

holds for each $\eta_{1} \in X_{1}$.

Proof Using Proposition 2.9 and Theorem 3.4, one finds that

$$
\begin{aligned}
\mathcal{L}_{2}\left(\eta_{1}+\eta_{2}\left(\eta_{1}\right)\right) & =m\left(\eta_{1}, \eta_{1}\right)+2 m\left(\eta_{1}, \eta_{2}\left(\eta_{1}\right)\right)+m\left(\eta_{2}\left(\eta_{1}\right), \eta_{2}\left(\eta_{1}\right)\right) \\
& =\mathcal{L}_{2}\left(\eta_{1}\right)+\mathcal{O}\left(\varepsilon\left\|\eta_{1}\right\|^{3}\right),
\end{aligned}
$$

and

$$
\mathcal{L}_{2}\left(\eta_{1}\right)=-\frac{3}{2} \eta_{1}^{2}+\underline{\mathcal{O}}\left(\varepsilon^{3 / 2}\left\|\mid \eta_{1}\right\|^{2}\right)
$$

because of (2.7) and Proposition 4.2. 
Lemma 4.4 The estimate

$$
\mathcal{N}\left(\eta_{1}+\eta_{2}\left(\eta_{1}\right)\right)=\mathcal{O}\left(\varepsilon\left\|\eta_{1}\right\|^{3}\right)
$$

holds for each $\eta_{1} \in X_{1}$.

Proof This result follows from Proposition 2.10(i) and Theorem 3.4.

We conclude that the reduced equation for $\eta_{1}$ is the perturbed full dispersion Korteweg-de Vries equation

$$
g(D) \eta_{1}+\varepsilon^{2} K_{0} \eta_{1}+\frac{3}{2} \chi_{0}(D) \eta_{1}^{2}+\underline{\mathcal{O}}_{0}\left(\varepsilon\left\|\eta_{1}\right\|^{2}\right)=0
$$

and applying Proposition 4.2, one can further simplify it to

$$
g(D) \eta_{1}+\varepsilon^{2} \eta_{1}+\frac{3}{2} \chi_{0}(D) \eta_{1}^{2}+\underline{\mathcal{O}}_{0}\left(\varepsilon\left\|\eta_{1}\right\|^{2}\right)+\underline{\mathcal{O}}_{0}\left(\varepsilon^{3}\left\|\eta_{1}\right\| \mid\right)=0 .
$$

Finally, we introduce the Korteweg-de Vries scaling

$$
\eta_{1}(x)=\varepsilon^{2} \rho(\varepsilon x)
$$

so that $\rho \in B_{R}(0) \subseteq \chi(\varepsilon D) H^{1}(\mathbb{R})$, where $R>0$ and $\varepsilon$ is chosen small enough that $\varepsilon^{3 / 2} R \leq R_{1}$, solves the equation

$$
\varepsilon^{-2} g(\varepsilon D) \rho+\rho+\frac{3}{2} \chi_{0}(\varepsilon D) \rho^{2}+\varepsilon^{1 / 2} \underline{\mathcal{O}}_{0}^{\varepsilon}\left(\|\rho\|_{1}\right)=0
$$

(note that $\|\eta\|=\varepsilon^{3 / 2}\|\rho\|_{1}$, the change of variable from $x$ to $X=\varepsilon x$ introduces an additional factor of $\varepsilon^{1 / 2}$ in the remainder term and the symbol $D$ now means $\left.-\mathrm{i} \partial_{X}\right)$. The invariance of the reduced equation under $\eta_{1}(x) \mapsto \eta_{1}(-x)$ is inherited by (4.1), which is invariant under the reflection $\rho(X) \mapsto \rho(-X)$.

\subsection{Weak Surface Tension}

In this section we compute the leading-order terms in the reduced equation (3.11) derived in Sect. 3.2. To this end, we write

$$
\eta_{1}=\eta_{1}^{+}+\eta_{1}^{-}
$$

where $\eta_{1}^{+}=\chi^{+}(D) \eta_{1}$ and $\eta_{1}^{-}=\overline{\eta_{1}^{+}}$, so that $\eta_{1}^{+}$satisfies the equation

$$
\begin{aligned}
& g(D) \eta_{1}^{+}+c_{0}^{2} \varepsilon^{2} K_{0} \eta_{1}^{+} \\
& \quad+\chi^{+}(D)\left(\mathcal{N}\left(\eta_{1}+F\left(\eta_{1}\right)+\eta_{3}\left(\eta_{1}\right)\right)+c_{0}^{2}\left(1-\varepsilon^{2}\right) \mathcal{L}_{2}\left(\eta_{1}\right)\right)=0
\end{aligned}
$$

(and $\eta_{1}^{-}$satisfies its complex conjugate). We again begin by showing how Fouriermultiplier operators acting upon the function $\eta_{1}$ may be approximated by constants. 


\section{Lemma 4.5 The estimates}

(i) $\partial_{x} \eta_{1}^{+}=+\mathrm{i} \omega \eta_{1}^{+}+\underline{\mathcal{O}}_{+}\left(\varepsilon\left\|\mid \eta_{1}\right\|\right)$,

(ii) $\partial_{x}^{2} \eta_{1}^{+}=-\omega^{2} \eta_{1}^{+}+\underline{\mathcal{O}}_{+}\left(\varepsilon\|\| \eta_{1}\|\|\right)$,

(iii) $K_{0} \eta_{1}^{+}=f(\omega) \eta_{1}^{+}+\underline{\mathcal{O}}_{+}\left(\varepsilon\left|\left\|\eta_{1}\right\|\right|\right)$,

(iv) $K_{0}\left(\left(\eta_{1}^{+}\right)^{2}\right)=f(2 \omega)\left(\eta_{1}^{+}\right)^{2}+\underline{\mathcal{O}}\left(\varepsilon^{3 / 2}\|\| \eta_{1} \|^{2}\right)$,

(v) $K_{0}\left(\eta_{1}^{+} \eta_{1}^{-}\right)=\eta_{1}^{+} \eta_{1}^{-}+\underline{\mathcal{O}}\left(\varepsilon^{3 / 2}\left\|\mid \eta_{1}\right\|^{2}\right)$,

(vi) $\mathcal{F}^{-1}\left[g(k)^{-1} \mathcal{F}\left[\left(\eta_{1}^{+}\right)^{2}\right]\right]=g(2 \omega)^{-1}\left(\eta_{1}^{+}\right)^{2}+\underline{\mathcal{O}}\left(\varepsilon^{3 / 2}\left\|\eta_{1}\right\|^{2}\right)$,

(vii) $\mathcal{F}^{-1}\left[g(k)^{-1} \mathcal{F}\left[\eta_{1}^{+} \eta_{1}^{-}\right]\right]=g(0)^{-1} \eta_{1}^{+} \eta_{1}^{-}+\underline{\mathcal{O}}\left(\varepsilon^{3 / 2}\left\|\eta_{1}\right\|^{2}\right)$,

(viii) $K_{0}\left(\left(\eta_{1}^{+}\right)^{2} \eta_{1}^{-}\right)=f(\omega)\left(\eta_{1}^{+}\right)^{2} \eta_{1}^{-}+\underline{\mathcal{O}}\left(\varepsilon^{2}\left\|\mid \eta_{1}\right\| \|^{3}\right)$

hold for each $\eta_{1} \in X_{1}$.

Proof Note that

$$
\left\|\partial_{x} \eta_{1}^{+}-\mathrm{i} \omega \eta_{1}^{+}\right\|_{0}^{2} \leq \int_{\mathbb{R}}(k-\omega)^{2}\left|\hat{\eta}_{1}(k)\right|^{2} \mathrm{~d} k \leq \varepsilon^{2}\left\|\left|\eta_{1} \|\right|^{2}\right.
$$

and iterating this argument yields (ii); moreover

$$
\left\|K_{0} \eta_{1}^{+}-f(\omega) \eta_{1}^{+}\right\|_{0}^{2}=\left\|(f(k)-f(\omega)) \hat{\eta}_{1}^{+}\right\|_{0} \lesssim \int_{\mathbb{R}}(k-\omega)^{2}\left|\hat{\eta}_{1}(k)\right|^{2} \mathrm{~d} k \leq \varepsilon^{2}\left\|\eta_{1}\right\| \|^{2} .
$$

The corresponding estimates for their derivatives are trivially satisfied since the operators are linear.

Notice that the quantities to be estimated in (iv)-(vii) are quadratic in $\eta_{1}$; it therefore suffices to estimate the corresponding bilinear operators. To this end we take $u_{1}$, $v_{1} \in \mathcal{X}_{1}$. The argument used for (iii) above yields

$$
\begin{aligned}
\| \mathcal{F} & {\left[K_{0}\left(u_{1}^{+} v_{1}^{+}\right)-f(2 \omega) u_{1}^{+} v_{1}^{+}\right] \|_{0} } \\
\lesssim & \left\|| k - 2 \omega | \int _ { \mathbb { R } } \left|\hat{u}_{1}^{+}(k-s)\left\|\hat{v}_{1}^{+}(s) \mid \mathrm{d} s\right\|_{0}\right.\right. \\
& \lesssim\left\|\int_{\mathbb{R}}|k-s-\omega|\left|\hat{u}_{1}^{+}(k-s) \| \hat{v}_{1}^{+}(s)\right| \mathrm{d} s\right. \\
& \quad+\int_{\mathbb{R}}|s-\omega|\left|\hat{u}_{1}^{+}(k-s)\left\|\hat{v}_{1}^{+}(s) \mid \mathrm{d} s\right\|_{0}\right. \\
& \left.\lesssim\left\||k-\omega| \hat{u}_{1}^{+}\right\|_{0}\left\|\hat{v}_{1}^{+}\right\|_{L^{1}(\mathbb{R})}+\left\|\hat{u}_{1}^{+}\right\|_{L^{1}(\mathbb{R})} \| \mid k-\omega, 0\right) \mid \hat{v}_{1}^{+} \|_{0} \\
& \lesssim \varepsilon^{3 / 2}\left\||| u_{1}\right\|\left|\left\|v_{1}\right\|\right|,
\end{aligned}
$$

where we have also used Young's inequality. Turning to (v), we note that

$$
\begin{aligned}
\| \mathcal{F} & {[K_{0}\left(u_{1}^{+} v_{1}^{-}\right)-\underbrace{f(0)}_{=1} u_{1}^{+} v_{1}^{-}] \|_{0} } \\
& \lesssim\left\|| k | \int _ { \mathbb { R } } \left|\hat{u}_{1}^{+}(k-s)\left\|\hat{v}_{1}^{-}(s) \mid \mathrm{d} s\right\|_{0}\right.\right.
\end{aligned}
$$




$$
\begin{aligned}
& \lesssim\left\|\int_{\mathbb{R}}\left|k-s-\omega\left\|\hat{u}_{1}^{+}(k-s)\right\| \hat{v}_{1}^{-}(s)\right| \mathrm{d} s\right. \\
& \quad+\int_{\mathbb{R}}|s+\omega|\left|\hat{u}_{1}^{+}(k-s)\left\|\hat{v}_{1}^{-}(s) \mid \mathrm{d} s\right\|_{0}\right. \\
& \lesssim\left\||k-\omega| \hat{u}_{1}^{+}\right\|\left\|_{0}\right\| \hat{v}_{1}^{+}\left\|_{L^{1}(\mathbb{R})}+\right\| \hat{u}_{1}^{+}\left\|_{L^{1}(\mathbb{R})}\right\||k+\omega| \hat{v}_{1}^{+} \|_{0} \\
& \lesssim \varepsilon^{3 / 2}\left\|\left|u _ { 1 } \left\|\left|\left\|v_{1}\right\|\right| .\right.\right.\right.
\end{aligned}
$$

Estimates (vi) and (vii) are obtained in the same fashion.

To establish (viii) we similarly estimate the relevant trilinear operator. Take $u_{1}, v_{1}$, $w_{1} \in \mathcal{X}_{1}$ and observe that

$$
\begin{aligned}
\| \mathcal{F} & {\left[K_{0}\left(u_{1}^{+} v_{1}^{+} w_{1}^{-}\right)-f(\omega) u_{1}^{+} v_{1}^{+} w_{1}^{-} \|_{0}\right.} \\
& \lesssim\left\||k-\omega| \int_{\mathbb{R}}\left|\hat{u}_{1}^{+}(k-s)\right|\left|\mathcal{F}\left[v_{1}^{+} w_{1}^{+}\right](s)\right| \mathrm{d} s_{1} \mathrm{~d} s_{2}\right\|_{0} \\
& \lesssim\left\|\int_{\mathbb{R}}\left|k-s+\omega \| \hat{u}_{1}^{+}(k-s)\right|\left|\mathcal{F}\left[v_{1}^{+} w_{1}^{+}\right](s)\right| \mathrm{d} s\right. \\
& \quad+\int_{\mathbb{R}} \mid\left(s-2 \omega|| \hat{u}_{1}^{+}(k-s)\left\|\mathcal{F}\left[v_{1}^{+} w_{1}^{+}\right](s) \mid \mathrm{d} s\right\|_{0}\right. \\
& \lesssim\left\||k+\omega| \hat{u}_{1}^{+}\right\|_{L^{1}(\mathbb{R})}\left\|v_{1}^{+} w_{1}^{+}\right\|_{0}+\left\|\hat{u}_{1}^{+}\right\|_{L^{1}(\mathbb{R})}\left\||k-2 \omega| \mathcal{F}\left[v_{1}^{+} w_{1}^{+}\right]\right\|_{0} \\
& \lesssim\left\||k+\omega| \hat{u}_{1}^{+}\right\|\left\|_{0}\right\| v_{1}^{+}\|\infty\| w_{1}^{+}\left\|_{0}+\right\| \hat{u}_{1}^{+}\left\|_{L^{1}(\mathbb{R})}\right\||k-2 \omega| \mathcal{F}\left[v_{1}^{+} w_{1}^{+}\right] \|_{0} \\
& \lesssim \varepsilon^{2}\|\| u_{1}\left\|\left|\left\|\left|\left\|v_{1}\right\|\right|\right\| w_{1}\|\| .\right.\right.
\end{aligned}
$$

We proceed by approximating each term in the quadratic and cubic parts of equation (4.2) according to the rules established in Lemma 4.5, recalling that

$$
\mathcal{N}\left(\eta_{1}+F\left(\eta_{1}\right)+\eta_{3}\right)=\mathcal{N}_{2}\left(\eta_{1}, \eta_{3}\right)-c_{0}^{2}\left(1-\varepsilon^{2}\right) \mathcal{N}_{1}\left(\eta_{1}, \eta_{3}\right),
$$

where $F\left(\eta_{1}\right), \mathcal{N}_{1}\left(\eta_{1}, \eta_{3}\right)$ and $\mathcal{N}_{1}\left(\eta_{1}, \eta_{3}\right)$ are defined by respectively (3.7), (3.9) and (3.10).

Proposition 4.6 The estimate

$F\left(\eta_{1}\right)=g(2 \omega)^{-1} c_{0}^{2} A(\omega)\left(\left(\eta_{1}^{+}\right)^{2}+\left(\eta_{1}^{-}\right)^{2}\right)+g(0)^{-1} c_{0}^{2} B(\omega) \eta_{1}^{+} \eta_{1}^{-}+\underline{\mathcal{O}}\left(\varepsilon^{3 / 2}\|\| \eta_{1} \|^{2}\right)$,

where

$$
A(\omega)=\frac{3}{2} \omega^{2}-\frac{1}{2} f(\omega)^{2}-f(\omega) f(2 \omega), \quad B(\omega)=\omega^{2}-2 f(\omega)-f(\omega)^{2},
$$

holds for each $\eta_{1} \in X_{1}$. 
Proof Using equation (2.7) and the expansions given in Lemma 4.5, we find that

$$
\mathcal{L}_{2}\left(\eta_{1}\right)=m\left(\eta_{1}, \eta_{1}\right)=A(\omega)\left(\left(\eta_{1}^{+}\right)^{2}+\left(\eta_{1}^{-}\right)^{2}\right)+B(\omega) \eta_{1}^{+} \eta_{1}^{-}+\underline{\mathcal{O}}\left(\varepsilon^{3 / 2}\left\|\eta_{1}\right\| \|^{2}\right)
$$

It follows that

$$
\begin{aligned}
& (1-\chi(D)) g(D)^{-1} \mathcal{L}_{2}\left(\eta_{1}\right) \\
& \quad=g(2 \omega)^{-1} A(\omega)\left(\left(\eta_{1}^{+}\right)^{2}+\left(\eta_{1}^{-}\right)^{2}\right)+g(0)^{-1} B(\omega) \eta_{1}^{+} \eta_{1}^{-}+\underline{\mathcal{O}}\left(\varepsilon^{3 / 2}\left\|\eta_{1}\right\| \|^{2}\right)
\end{aligned}
$$

because of Lemma 4.5(vi), (vii) and the facts that $\chi(D) \mathcal{L}_{2}\left(\eta_{1}\right)=0$ and

$$
(1-\chi(D)) g(D)^{-1} \underline{\mathcal{O}}\left(\varepsilon^{3 / 2}\left\|\eta_{1}\right\|^{2}\right)=\underline{\mathcal{O}}\left(\varepsilon^{3 / 2}\left\|\eta_{1}\right\|^{2}\right)
$$

(since $(1-\chi(k)) g(k)^{-1}$ is bounded). We conclude that

$$
\begin{aligned}
F\left(\eta_{1}\right) & =c_{0}^{2}\left(1-\varepsilon^{2}\right)(1-\chi(D)) g(D)^{-1} \mathcal{L}_{2}\left(\eta_{1}\right) \\
& =g(2 \omega)^{-1} c_{0}^{2} A(\omega)\left(\left(\eta_{1}^{+}\right)^{2}+\left(\eta_{1}^{-}\right)^{2}\right)+g(0)^{-1} c_{0}^{2} B(\omega) \eta_{1}^{+} \eta_{1}^{-}+\underline{\mathcal{O}}\left(\varepsilon^{3 / 2}\left\|\eta_{1}\right\|^{2}\right) .
\end{aligned}
$$

\section{Proposition 4.7 The estimate}

$$
\begin{aligned}
& \chi^{+}(D) \mathcal{N}_{1}\left(\eta_{1}, \eta_{3}\left(\eta_{1}\right)\right) \\
& \quad=\left(2 g(2 \omega)^{-1} A(\omega)^{2} c_{0}^{2}+g(0)^{-1} B(\omega)^{2} c_{0}^{2}\right) \chi^{+}(D)\left(\left(\eta_{1}^{+}\right)^{2} \eta_{1}^{-}\right)+\underline{\mathcal{O}}_{+}\left(\varepsilon^{3 / 2}\|\| \eta_{1} \|^{3}\right)
\end{aligned}
$$

holds for each $\eta_{1} \in X_{1}$.

Proof Observe that

$$
\begin{aligned}
\mathcal{N}_{1}\left(\eta_{1}, \eta_{3}\left(\eta_{1}\right)\right) & =2 m\left(\eta_{1}, F\left(\eta_{1}\right)+\eta_{3}\left(\eta_{1}\right)\right)+m\left(F\left(\eta_{1}\right)+\eta_{3}\left(\eta_{1}\right), F\left(\eta_{1}\right)+\eta_{3}\left(\eta_{1}\right)\right) \\
& =2 m\left(\eta_{1}, F\left(\eta_{1}\right)\right)+\mathcal{O}\left(\varepsilon^{3 / 2}\|\| \eta_{1}\|\|^{3}\right),
\end{aligned}
$$

in which we have used the calculations

$$
m\left(\eta_{1}, \eta_{3}\left(\eta_{1}\right)\right)=\mathcal{O}\left(\varepsilon^{3 / 2}\left\|\eta_{1}\right\|^{3}\right), \quad m\left(F\left(\eta_{1}\right), \eta_{3}\left(\eta_{1}\right)\right)=\mathcal{O}\left(\varepsilon^{3 / 2}\left\|\eta_{1}\right\| \|^{4}\right)
$$

(see Propositions 2.9 and 3.5 and Theorem 3.10) and

$$
m\left(F\left(\eta_{1}\right), F\left(\eta_{1}\right)\right)=\underline{\mathcal{O}}\left(\varepsilon^{3 / 2}\left\|\eta_{1}\right\|^{4}\right)
$$

(because of Propositions 2.9 and 4.6). Furthermore 


$$
\begin{aligned}
\chi^{+}(D) m\left(\eta_{1}, F\left(\eta_{1}\right)\right)= & g(2 \omega)^{-1} c_{0}^{2} A(\omega) \chi^{+}(D) m\left(\eta_{1}^{-},\left(\eta_{1}^{+}\right)^{2}\right) \\
& +g(0)^{-1} c_{0}^{2} B(\omega) \chi^{+}(D) m\left(\eta_{1}^{+}, \eta_{1}^{+} \eta_{1}^{-}\right)+\underline{\mathcal{O}}_{+}\left(\varepsilon^{3 / 2}\left\|\eta_{1}\right\| \|^{3}\right),
\end{aligned}
$$

and it follows from (2.7) and Lemma 4.5 that

$$
\begin{aligned}
\left.m\left(\eta_{1}^{-},\left(\eta_{1}^{+}\right)^{2}\right)\right) & =A(\omega)\left(\eta_{1}^{+}\right)^{2} \eta_{1}^{-}+\underline{\mathcal{O}}\left(\left.\varepsilon^{3 / 2}\left\|\eta_{1}\right\|\right|^{3}\right), \\
m\left(\eta_{1}^{+}, \eta_{1}^{+} \eta_{1}^{-}\right) & =\frac{1}{2} B(\omega)\left(\eta_{1}^{+}\right)^{2} \eta_{1}^{-}+\underline{\mathcal{O}}\left(\varepsilon^{3 / 2}\left\|\mid \eta_{1}\right\|^{3}\right) .
\end{aligned}
$$

\section{Proposition 4.8 The estimates}

$$
\begin{aligned}
& \chi^{+}(D) \mathcal{K}_{3}\left(\eta_{1}+F\left(\eta_{1}\right)+\eta_{3}\left(\eta_{1}\right)\right)=-\frac{3}{2} \beta \omega^{4} \chi^{+}(D)\left(\left(\eta_{1}^{+}\right)^{2} \eta_{1}^{-}\right)+\underline{\mathcal{O}}_{+}\left(\varepsilon^{3 / 2}\left\|\eta_{1}\right\|^{3}\right), \\
& \chi^{+}(D) \mathcal{L}_{3}\left(\eta_{1}+F\left(\eta_{1}\right)+\eta_{3}\left(\eta_{1}\right)\right)=C(\omega) \chi^{+}(D)\left(\left(\eta_{1}^{+}\right)^{2} \eta_{1}^{-}\right)+\underline{\mathcal{O}}_{+}\left(\varepsilon^{3 / 2}\left\|\eta_{1}\right\| \|^{3}\right),
\end{aligned}
$$

where

$$
C(\omega)=-6 \omega^{2} f(\omega)+4 f(\omega)^{2}+2 f(\omega)^{2} f(2 \omega),
$$

hold for each $\eta_{1} \in X_{1}$.

Proof Using the estimates for $F\left(\eta_{1}\right)$ and $\eta_{3}\left(\eta_{1}\right)$ given in Proposition 3.5 and Theorem 3.10 , we find that

$$
\mathcal{K}_{3}\left(\eta_{1}+F\left(\eta_{1}\right)+\eta_{3}\left(\eta_{1}\right)\right)=\mathcal{K}_{3}\left(\eta_{1}\right)+\mathcal{O}\left(\varepsilon^{3 / 2}\left\|\eta_{1}\right\|^{4}\right)
$$

and

$$
\chi^{+}(D) \mathcal{K}_{3}\left(\eta_{1}\right)=-\frac{3}{2} \beta \omega^{4} \chi^{+}(D)\left(\left(\eta_{1}^{+}\right)^{2} \eta_{1}^{-}\right)+\underline{\mathcal{O}}_{+}\left(\varepsilon^{3 / 2}\left\|\eta_{1}\right\|^{3}\right)
$$

(because of equation (2.5)). It similarly follows from the formula

$$
\mathcal{L}_{3}(\eta)=-K_{0} \eta K_{1}(\eta) \eta-\eta_{x}^{2} K_{0} \eta+m_{2}(\eta, \eta) \eta
$$

(see equation (2.6) and Remark 2.5) that

$$
\mathcal{L}_{3}\left(\eta_{1}+F\left(\eta_{1}\right)+\eta_{3}\left(\eta_{1}\right)\right)=\mathcal{L}_{3}\left(F\left(\eta_{1}\right)+\eta_{1}\right)+\mathcal{O}\left(\varepsilon^{3 / 2}\left\|\eta_{1}\right\|^{4}\right),
$$

and using Corollary 2.8(ii) twice yields

$$
\mathcal{L}_{3}\left(F\left(\eta_{1}\right)+\eta_{1}\right)=\mathcal{L}_{3}\left(\eta_{1}\right)+\underline{\mathcal{O}}\left(\varepsilon^{3 / 2} \mid\left\|\eta_{1}\right\|^{3}\right)
$$

and

$$
\chi^{+}(D) \mathcal{L}_{3}\left(\eta_{1}\right)=C(\omega) \chi^{+}(D)\left(\left(\eta_{1}^{+}\right)^{2} \eta_{1}^{-}\right)+\underline{\mathcal{O}}_{+}\left(\varepsilon^{3 / 2}\left\|\eta_{1}\right\|^{3}\right) .
$$


Proposition 4.9 The estimates

$$
\begin{aligned}
& \mathcal{K}_{\mathrm{r}}\left(\eta_{1}+F\left(\eta_{1}\right)+\eta_{3}\left(\eta_{1}\right)\right)=\mathcal{O}\left(\varepsilon^{2}\left\|\eta_{1}\right\|^{5}\right), \\
& \mathcal{L}_{\mathrm{r}}\left(\eta_{1}+F\left(\eta_{1}\right)+\eta_{3}\left(\eta_{1}\right)\right)=\mathcal{O}\left(\varepsilon^{3 / 2}\left\|\eta_{1}\right\|^{4}\right)
\end{aligned}
$$

hold for each $\eta_{1} \in X_{1}$.

Proof This result follows from Propositions 2.10(ii) and 3.5 and Theorem 3.10.

Proposition 4.10 The estimate

$$
-\frac{1}{2} \chi^{+}(D)\left(K_{1}\left(\eta_{1}+F\left(\eta_{1}\right)+\eta_{3}\left(\eta_{1}\right)\right)\left(\eta_{1}+F\left(\eta_{1}\right)+\eta_{3}\left(\eta_{1}\right)\right)^{2}=\underline{\mathcal{O}}_{+}\left(\varepsilon^{3 / 2}\left\|\eta_{1}\right\| \|^{4}\right)\right.
$$

holds for each $\eta_{1} \in X_{1}$.

Proof Using Proposition 3.5 and Theorem 3.10 we find that

$$
\begin{aligned}
-\frac{1}{2}\left(K _ { 1 } \left(\eta_{1}\right.\right. & \left.+F\left(\eta_{1}\right)+\eta_{3}\left(\eta_{1}\right)\right)\left(\eta_{1}+F\left(\eta_{1}\right)+\eta_{3}\left(\eta_{1}\right)\right)^{2} \\
& =-\frac{1}{2}\left(K_{1}\left(\eta_{1}\right) \eta_{1}\right)^{2}+\mathcal{O}\left(\varepsilon^{3 / 2}\left\|\eta_{1}\right\|^{4}\right),
\end{aligned}
$$

and furthermore

$$
-\frac{1}{2} \chi^{+}(D)\left(K_{1}\left(\eta_{1}\right) \eta_{1}\right)^{2}=-\frac{1}{2} \chi^{+}(D)\left(\left(\eta_{1} \eta_{1 x}\right)_{x}+K_{0}\left(\eta_{1} K_{0} \eta_{1}\right)\right)^{2}=0
$$

(see Lemma 2.6(i)).

Corollary 4.11 The estimate

$$
\chi^{+}(D) \mathcal{N}_{2}\left(\eta_{1}, \eta_{3}\left(\eta_{1}\right)\right)=-\frac{3}{2} \beta \omega^{4}-C(\omega) c_{0}^{2}+\underline{\mathcal{O}}_{+}\left(\varepsilon^{3 / 2}\left\|\eta_{1}\right\| \|^{3}\right)
$$

holds for each $\eta_{1} \in X_{1}$.

We conclude that the reduced equation for $\eta_{1}$ is the perturbed full dispersion nonlinear Schrödinger equation

$$
g(D) \eta_{1}^{+}+c_{0}^{2} \varepsilon^{2} K_{0} \eta_{1}^{+}-D(\omega) \chi^{+}\left(\left|\eta_{1}^{+}\right|^{2} \eta_{1}^{+}\right)+\underline{\mathcal{O}}_{+}\left(\left.\varepsilon^{3 / 2}\left\|\eta_{1}\right\|\right|^{3}\right)=0,
$$

where

$$
D(\omega)=2 g(2 \omega)^{-1} A(\omega)^{2} c_{0}^{4}+2 g(0)^{-1} B(\omega)^{2} c_{0}^{4}+C(\omega) c_{0}^{2}+\frac{3}{2} \beta \omega^{4},
$$

and applying Lemma 4.5(iii), one can further simplify it to

$$
\begin{aligned}
& g(D) \eta_{1}^{+}+c_{0}^{2} f(\omega) \varepsilon^{2} \eta_{1}^{+} \\
& \quad-D(\omega) \chi^{+}\left(\left|\eta_{1}^{+}\right|^{2} \eta_{1}^{+}\right)+\underline{\mathcal{O}}_{+}\left(\varepsilon^{3 / 2}\left\|\left|\eta_{1} \|\right|^{3}\right)+\underline{\mathcal{O}}_{+}\left(\varepsilon^{3}\left\|\left|\eta_{1} \|\right|\right)=0 .\right.\right.
\end{aligned}
$$


Finally, we introduce the nonlinear Schrödinger scaling

$$
\eta_{1}^{+}(x)=\frac{1}{2} \varepsilon \zeta(\varepsilon x) \mathrm{e}^{\mathrm{i} \omega x},
$$

so that $\zeta \in B_{R}(0) \subseteq \chi_{0}(\varepsilon D) H^{1}(\mathbb{R})$, where $R>0$ and $\varepsilon$ is chosen small enough that $\varepsilon^{1 / 2} R \leq 2 R_{1}$, solves the equation

$$
\varepsilon^{-2} g(\omega+\varepsilon D) \zeta+c_{0}^{2} f(\omega) \zeta-\frac{1}{4} D(\omega) \chi_{0}(\varepsilon D)\left(|\zeta|^{2} \zeta\right)+\varepsilon^{1 / 2} \underline{\mathcal{O}}_{0}^{\varepsilon}\left(\|\zeta\|_{1}\right)=0
$$

(note that $\left\|\eta_{1}\right\|=\varepsilon^{1 / 2}\|\zeta\|_{1}$, the change of variable from $x$ to $X=\varepsilon x$ introduces an additional factor of $\varepsilon^{1 / 2}$ in the remainder term and the symbol $D$ now means $-\mathrm{i} \partial_{X}$ ). The invariance of the reduced equation under $\eta_{1}(x) \mapsto \eta_{1}(-x)$ is inherited by (4.3), which is invariant under the reflection $\zeta(X) \mapsto \overline{\zeta(-X)}$.

\section{Solution of the Reduced Equation}

In this section, we find solitary-wave solutions of the reduced equations

$$
\varepsilon^{-2} g(\varepsilon D) \rho+\rho+\frac{3}{2} \chi_{0}(\varepsilon D) \rho^{2}+\varepsilon^{1 / 2} \underline{\mathcal{O}}_{0}^{\varepsilon}\left(\|\rho\|_{1}\right)=0
$$

and

$$
\varepsilon^{-2} g(\omega+\varepsilon D) \zeta+c_{0}^{2} f(\omega) \zeta-a_{3} \chi_{0}(\varepsilon D)\left(|\zeta|^{2} \zeta\right)+\varepsilon^{1 / 2} \underline{\mathcal{O}}_{0}^{\varepsilon}\left(\|\zeta\|_{1}\right)=0
$$

noting that in the formal limit $\varepsilon \rightarrow 0$ they reduce to respectively the stationary Korteweg-de Vries equation

$$
-\left(\beta-\frac{1}{3}\right) \rho_{X X}+\rho+\frac{3}{2} \rho^{2}=0
$$

and the stationary nonlinear Schrödinger equation

$$
-a_{1} \zeta_{X X}+a_{2} \zeta-a_{3}|\zeta|^{2} \zeta=0
$$

which have explicit (symmetric) solitary-wave solutions $\rho^{\star}$ and $\pm \zeta^{\star}$ (Eqs. (1.7) and (1.10)). For this purpose we use a perturbation argument, rewriting (5.1) and (5.2) as fixed-point equations and applying the following version of the implicit-function theorem.

Theorem 5.1 Let $\mathcal{X}$ be a Banach space, $X_{0}$ and $\Lambda_{0}$ be open neighbourhoods of respectively $x^{\star}$ in $\mathcal{X}$ and the origin in $\mathbb{R}$ and $\mathcal{G}: X_{0} \times \Lambda_{0} \rightarrow \mathcal{X}$ be a function which is differentiable with respect to $x \in X_{0}$ for each $\lambda \in \Lambda_{0}$. Furthermore, suppose that $\mathcal{G}\left(x^{\star}, 0\right)=0, \mathrm{~d}_{1} \mathcal{G}\left[x^{\star}, 0\right]: \mathcal{X} \rightarrow \mathcal{X}$ is an isomorphism,

$$
\lim _{x \rightarrow x^{\star}}\left\|\mathrm{d}_{1} \mathcal{G}[x, 0]-\mathrm{d}_{1} \mathcal{G}\left[x^{\star}, 0\right]\right\|_{\mathcal{L}(\mathcal{X})}=0
$$


and

$$
\lim _{\lambda \rightarrow 0}\|\mathcal{G}(x, \lambda)-\mathcal{G}(x, 0)\| \mathcal{X}=0, \quad \lim _{\lambda \rightarrow 0}\left\|\mathrm{~d}_{1} \mathcal{G}[x, \lambda]-\mathrm{d}_{1} \mathcal{G}[x, 0]\right\|_{\mathcal{L}(\mathcal{X})}=0
$$

uniformly over $x \in X_{0}$.

There exist open neighbourhoods $X$ of $x^{\star}$ in $\mathcal{X}$ and $\Lambda$ of 0 in $\mathbb{R}$ (with $X \subseteq X_{0}$, $\Lambda \subseteq \Lambda_{0}$ ) and a uniquely determined mapping $h: \Lambda \rightarrow X$ with the properties that

(i) $h$ is continuous at the origin (with $\left.h(0)=x^{\star}\right)$,

(ii) $\mathcal{G}(h(\lambda), \lambda)=0$ for all $\lambda \in \Lambda$,

(iii) $x=h(\lambda)$ whenever $(x, \lambda) \in X \times \Lambda$ satisfies $\mathcal{G}(x, \lambda)=0$.

\subsection{Strong Surface Tension}

Theorem 5.2 For each sufficiently small value of $\varepsilon>0$ equation (5.1) has a smallamplitude, symmetric solution $\rho_{\varepsilon}$ in $\chi_{0}(\varepsilon D) H^{1}(\mathbb{R})$ with $\left\|\rho_{\varepsilon}-\rho^{\star}\right\|_{1} \rightarrow 0$ as $\varepsilon \rightarrow 0$.

The first step in the proof of Theorem 5.2 is to write (5.1) as the fixed-point equation

$$
\rho+\varepsilon^{2}\left(\varepsilon^{2}+g(\varepsilon D)\right)^{-1}\left(\frac{3}{2} \chi_{0}(\varepsilon D) \rho^{2}+\varepsilon^{1 / 2} \underline{\mathcal{O}}_{0}^{\varepsilon}\left(\|\rho\|_{1}\right)\right)=0
$$

and use the following result to 'replace' the nonlocal operator with a differential operator.

Proposition 5.3 The inequality

$$
\left|\frac{\varepsilon^{2}}{\varepsilon^{2}+g(\varepsilon k)}-\frac{1}{1+\left(\beta-\frac{1}{3}\right) k^{2}}\right| \lesssim \frac{\varepsilon}{\left(1+k^{2}\right)^{1 / 2}}
$$

holds uniformly over $|k|<\delta / \varepsilon$.

Proof Clearly

$$
\left|\frac{\varepsilon^{2}}{\varepsilon^{2}+g(\varepsilon k)}-\frac{1}{1+\left(\beta-\frac{1}{3}\right) k^{2}}\right|=\frac{g(\varepsilon k)-\left(\beta-\frac{1}{3}\right) k^{2} \varepsilon^{2}}{\left(\varepsilon^{2}+g(\varepsilon k)\right)\left(1+\left(\beta-\frac{1}{3}\right) k^{2}\right)} ;
$$

furthermore

$$
g(s)-\left(\beta-\frac{1}{3}\right) s^{2} \lesssim|s|^{3}, \quad|s| \leq \delta,
$$

and

$$
g(s) \gtrsim s^{2}, \quad s \in \mathbb{R} .
$$


It follows that

$$
\left|\frac{\varepsilon^{2}}{\varepsilon^{2}+g(\varepsilon k)}-\frac{1}{1+\left(\beta-\frac{1}{3}\right) k^{2}}\right| \lesssim \frac{\varepsilon|k|^{3}}{\left(1+|k|^{2}\right)^{2}}, \quad|k|<\delta / \varepsilon .
$$

Using the above proposition, one can write equation (5.5) as

$$
\rho+F_{\varepsilon}(\rho)=0
$$

where

$$
F_{\varepsilon}(\rho)=\frac{3}{2}\left(1-\left(\beta-\frac{1}{3}\right) \partial_{X}^{2}\right)^{-1} \chi_{0}(\varepsilon D) \rho^{2}+\varepsilon^{1 / 2} \underline{\mathcal{O}}_{1}^{\varepsilon}\left(\|\rho\|_{1}\right)
$$

It is convenient to replace this equation with

$$
\rho+\tilde{F}_{\varepsilon}(\rho)=0
$$

where $\tilde{F}_{\varepsilon}(\rho)=F_{\varepsilon}\left(\chi_{0}(\varepsilon D) \rho\right)$ and study it in the fixed space $H^{1}(\mathbb{R})$ (the solution sets of the two equations evidently coincide).

We establish Theorem 5.6 by applying Theorem 5.1 with

$$
\mathcal{X}=H_{\mathrm{e}}^{1}(\mathbb{R}):=\left\{u \in H^{1}(\mathbb{R}): u(X)=u(-X) \text { for all } X \in \mathbb{R}\right\}
$$

$X=B_{R}(0), \Lambda_{0}=\left(-\varepsilon_{0}, \varepsilon_{0}\right)$ for a sufficiently small value of $\varepsilon_{0}$, and

$$
\mathcal{G}(\rho, \varepsilon):=\rho+\tilde{F}_{|\varepsilon|}(\rho)
$$

(here $\varepsilon$ is replaced by $|\varepsilon|$ so that $\mathcal{G}(\rho, \varepsilon)$ is defined for $\varepsilon$ in a full neighbourhood of the origin in $\mathbb{R}$ ). Observe that

$$
\begin{aligned}
& \mathcal{G}(\rho, \varepsilon)-\mathcal{G}(\rho, 0) \\
& \quad=\frac{3}{2}\left(1-\left(\beta-\frac{1}{3}\right) \partial_{X}^{2}\right)^{-1}\left[\chi_{0}(|\varepsilon| D)\left(\chi_{0}(|\varepsilon| D) \rho\right)^{2}-\rho^{2}\right]+|\varepsilon|^{1 / 2} \underline{\mathcal{O}}_{1}^{|\varepsilon|}\left(\|\rho\|_{1}\right),
\end{aligned}
$$

and noting that

$$
\lim _{\varepsilon \rightarrow 0}\left\|\chi_{0}(|\varepsilon| D)-I\right\|_{\mathcal{L}\left(H^{1}(\mathbb{R}), H^{3 / 4}(\mathbb{R})\right)}=0
$$

because 


$$
\begin{aligned}
\left\|\chi_{0}(|\varepsilon| D) u-u\right\|_{3 / 4}^{2} & =\int_{|k|>\frac{\delta}{|\varepsilon|}}\left(1+|k|^{2}\right)^{3 / 4}|\hat{u}|^{2} \mathrm{~d} k \\
& \leq \sup _{|k|>\frac{\delta}{|\varepsilon|}}\left(1+|k|^{2}\right)^{-1 / 4} \int_{|k|>\frac{\delta}{|\varepsilon|}}\left(1+|k|^{2}\right)|\hat{u}|^{2} \mathrm{~d} k \\
& \leq\left(1+\frac{\delta^{2}}{|\varepsilon|^{2}}\right)^{-1 / 4}\|u\|_{1}^{2},
\end{aligned}
$$

that

$$
\begin{aligned}
\chi_{0}(|\varepsilon| D) & \left(\chi_{0}(|\varepsilon| D) \rho\right)^{2}-\rho^{2} \\
& =\chi_{0}(|\varepsilon| D)\left(\chi_{0}(|\varepsilon| D) \rho+\rho\right)\left(\chi_{0}(|\varepsilon| D)-I\right) \rho+\left(\chi_{0}(|\varepsilon| D)-I\right) \rho^{2}
\end{aligned}
$$

and that $H^{3 / 4}(\mathbb{R})$ is a Banach algebra, we find that

$$
\lim _{\varepsilon \rightarrow 0}\|\mathcal{G}(\rho, \varepsilon)-\mathcal{G}(\rho, 0)\|_{1}=0, \quad \lim _{\varepsilon \rightarrow 0}\left\|\mathrm{~d}_{1} \mathcal{G}[\rho, \varepsilon]-\mathrm{d}_{1} \mathcal{G}[\rho, 0]\right\|_{\mathcal{L}\left(H^{1}(\mathbb{R})\right)}=0
$$

uniformly over $\rho \in B_{R}(0)$. The equation

$$
\mathcal{G}(\rho, 0)=\rho+\frac{3}{2}\left(1-\left(\beta-\frac{1}{3}\right) \partial_{X}^{2}\right)^{-1} \rho^{2}=0
$$

has the (unique) nontrivial solution $\rho^{\star}$ in $H_{\mathrm{e}}^{1}(\mathbb{R})$ and it remains to show that

$$
\mathrm{d}_{1} \mathcal{G}\left[\rho^{\star}, 0\right]=I+3\left(1-\left(\beta-\frac{1}{3}\right) \partial_{X}^{2}\right)^{-1}\left(\rho^{\star} \cdot\right)
$$

is an isomorphism.

Noting that $\rho^{\star} \in \mathcal{S}(\mathbb{R})$, we obtain the following result by a familiar argument (see Kirchgässner [17, Proposition 5.1] or Friesecke \& Pego [10, §4]).

Proposition 5.4 The formula $\rho \mapsto 3\left(1-\left(\beta-\frac{1}{3}\right) \partial_{X}^{2}\right)^{-1}\left(\rho^{\star} \rho\right)$ defines a compact linear operator $H^{1}(\mathbb{R}) \rightarrow H^{1}(\mathbb{R})$ and $H_{\mathrm{e}}^{1}(\mathbb{R}) \rightarrow H_{\mathrm{e}}^{1}(\mathbb{R})$.

This proposition implies in particular that $\mathrm{d}_{1} \mathcal{G}\left[\rho^{\star}, 0\right]$ is a Fredholm operator with index 0 . Its kernel coincides with the set of symmetric bounded solutions of the ordinary differential equation

$$
-\left(\beta-\frac{1}{3}\right) \rho_{X X}+\rho+3 \rho^{\star} \rho=0,
$$

and the next proposition shows that this set consists of only the trivial solution, so that $\mathrm{d}_{1} \mathcal{G}\left[\rho^{\star}, 0\right]$ is an isomorphism.

Proposition 5.5 Every bounded solution to the equation (5.6) is a multiple of $\rho_{X}^{\star}$ and is therefore antisymmetric. 
Proof Define

$$
\begin{aligned}
& \rho_{1}(\check{X})=\operatorname{sech}^{2}\left(\frac{1}{2} \check{X}\right) \tanh \left(\frac{1}{2} \check{X}\right), \\
& \rho_{2}(\check{X})=\cosh \check{X}+\frac{3}{2} \operatorname{sech}^{2}\left(\frac{1}{2} \check{X}\right)\left(-8+2 \cosh \check{X}+5 \check{X} \tanh \left(\frac{1}{2} \check{X}\right)\right),
\end{aligned}
$$

where $\check{X}=\left(\beta-\frac{1}{3}\right)^{-1 / 2} X$, and observe that $\left\{\rho_{1}, \rho_{2}\right\}$ is a fundamental solution set for (5.6). Its bounded solutions are therefore precisely the multiples of $\rho_{1}$.

\subsection{Weak Surface Tension}

Theorem 5.6 For each sufficiently small value of $\varepsilon>0$ equation (5.2) has two smallamplitude, symmetric solutions $\zeta_{\varepsilon}^{ \pm}$in $\chi_{0}(\varepsilon D) H^{1}(\mathbb{R})$ with $\left\|\zeta_{\varepsilon}^{ \pm} \mp \zeta^{\star}\right\|_{1} \rightarrow 0$ as $\varepsilon \rightarrow 0$.

We again begin the proof of Theorem 5.6 by 'replacing' the nonlocal operator in the fixed-point formulation

$$
\zeta+\varepsilon^{2}\left(\varepsilon^{2} c_{0}^{2} f(\omega)+g(\omega+\varepsilon D)\right)^{-1}\left(-a_{3} \chi_{0}(\varepsilon D)\left(|\zeta|^{2} \zeta\right)+\varepsilon^{1 / 2} \underline{\mathcal{O}}_{0}^{\varepsilon}\left(\|\zeta\|_{1}\right)\right)=0
$$

of equation (5.2) with a differential operator.

Proposition 5.7 The inequality

$$
\left|\frac{\varepsilon^{2}}{c_{0}^{2} f(\omega) \varepsilon^{2}+g(\omega+\varepsilon k)}-\frac{1}{a_{1}+a_{2} k^{2}}\right| \lesssim \frac{\varepsilon}{\left(1+k^{2}\right)^{1 / 2}}
$$

holds uniformly over $|k|<\delta / \varepsilon$.

Proof Clearly

$$
\begin{aligned}
& \left|\frac{\varepsilon^{2}}{c_{0}^{2} f(\omega) \varepsilon^{2}+g(\omega+\varepsilon k)}-\frac{1}{a_{1}+a_{2} k^{2}}\right| \\
& =\frac{\left|g(\omega+\varepsilon k)-\varepsilon^{2}\left(c_{0}^{2} f(\omega)+\frac{1}{2} g^{\prime \prime}(\omega) k^{2}\right)\right|}{\left(c_{0}^{2} f(\omega) \varepsilon^{2}+g(\omega+\varepsilon k)\right)\left(c_{0}^{2} f(\omega)+\frac{1}{2} g^{\prime \prime}(\omega) k^{2}\right)},
\end{aligned}
$$

while

$$
g(\omega+s)-\frac{1}{2} g^{\prime \prime}(\omega) s^{2} \lesssim|s|^{3}, \quad|s| \leq \delta,
$$

and

$$
g(\omega+s) \gtrsim s^{2}, \quad s \in \mathbb{R} .
$$


It follows that

$$
\left|\frac{\varepsilon^{2}}{c_{0}^{2} f(\omega) \varepsilon^{2}+g(\omega+\varepsilon k)}-\frac{1}{a_{1}+a_{2} k^{2}}\right| \lesssim \frac{\varepsilon|k|^{3}}{\left(1+|k|^{2}\right)^{2}}, \quad|k|<\delta / \varepsilon
$$

Using the above proposition, one can write equation (5.2) as

$$
\zeta+F_{\varepsilon}(\zeta)=0
$$

where

$$
F_{\varepsilon}(\zeta)=-a_{3}\left(a_{1}-a_{2} \partial_{X}^{2}\right)^{-1}|\zeta|^{2} \zeta+\varepsilon^{1 / 2} \underline{\mathcal{O}}_{1}^{\varepsilon}\left(\|\zeta\|_{1}\right)
$$

or equivalently with

$$
\zeta+\tilde{F}_{\varepsilon}(\zeta)=0
$$

where $\tilde{F}_{\varepsilon}(\zeta)=F_{\varepsilon}\left(\chi_{0}(\varepsilon D) \zeta\right)$, and studying it in the fixed space $H^{1}(\mathbb{R}, \mathbb{C})$. We establish Theorem 5.2 by applying Theorem 5.1 with

$$
\mathcal{X}=H_{\mathrm{e}}^{1}(\mathbb{R}, \mathbb{C})=\left\{\zeta \in H^{1}(\mathbb{R}): \zeta(X)=\overline{\zeta(-X)} \text { for all } X \in \mathbb{R}\right\}
$$

$X=B_{R}(0), \Lambda_{0}=\left(-\varepsilon_{0}, \varepsilon_{0}\right)$ for a sufficiently small value of $\varepsilon_{0}$ and

$$
\mathcal{G}(\zeta, \varepsilon):=\zeta+\tilde{F}_{|\varepsilon|}(\zeta)
$$

Observe that

$$
\begin{aligned}
\mathcal{G}(\zeta, \varepsilon) & -\mathcal{G}(\zeta, 0) \\
=- & \left.a_{3}\left(a_{1}-a_{2} \partial_{X}^{2}\right)^{-1}\left[\chi_{0}(|\varepsilon| D)\left(\left|\chi_{0}(|\varepsilon| D) \zeta\right|^{2} \chi_{0}(|\varepsilon| D) \zeta\right)-|\zeta|^{2} \zeta \mid\right)\right] \\
& +|\varepsilon|^{\frac{1}{2}} \underline{\mathcal{O}}_{1}^{|\varepsilon|}\left(\|\zeta\|_{1}\right),
\end{aligned}
$$

so that

$$
\lim _{\varepsilon \rightarrow 0}\|\mathcal{G}(\zeta, \varepsilon)-\mathcal{G}(\zeta, 0)\|_{1}=0, \quad \lim _{\varepsilon \rightarrow 0}\left\|\mathrm{~d}_{1} \mathcal{G}[\zeta, \varepsilon]-\mathrm{d}_{1} \mathcal{G}[\zeta, 0]\right\|_{\mathcal{L}\left(H^{1}(\mathbb{R}, \mathbb{C})\right)}=0
$$

uniformly over $\zeta \in B_{R}(0)$.

The equation

$$
\mathcal{G}(\zeta, 0)=\zeta-a_{3}\left(a_{1}-a_{2} \partial_{X}^{2}\right)^{-1}|\zeta|^{2} \zeta=0
$$


has (precisely two) nontrivial solutions $\pm \zeta^{\star}$ in $H_{\mathrm{e}}^{1}(\mathbb{R}, \mathbb{C}$ ), which are both real, and the fact that $\mathrm{d}_{1} \mathcal{G}\left[ \pm \zeta^{\star}, 0\right]$ is an isomorphism is conveniently established by using real coordinates. Define $\zeta_{1}=\operatorname{Re} \zeta$ and $\zeta_{2}=\operatorname{Im} \zeta$, so that

$$
\mathrm{d}_{1} \mathcal{G}\left[ \pm \zeta^{\star}, 0\right]\left(\zeta_{1}+\mathrm{i} \zeta_{2}\right)=\mathcal{G}_{1}\left(\zeta_{1}\right)+\mathrm{i} \mathcal{G}_{2}\left(\zeta_{2}\right)
$$

where $\mathcal{G}_{1}: H_{\mathrm{e}}^{1}(\mathbb{R}) \rightarrow H_{\mathrm{e}}^{1}(\mathbb{R})$ and $\mathcal{G}_{2}: H_{\mathrm{o}}^{1}(\mathbb{R}) \rightarrow H_{\mathrm{o}}^{1}(\mathbb{R})$ are given by

$$
\begin{aligned}
& \mathcal{G}_{1}\left(\zeta_{1}\right)=\zeta_{1}-3 a_{3}\left(a_{1}-a_{2} \partial_{X}^{2}\right)^{-1} \zeta^{\star 2} \zeta_{1}, \\
& \mathcal{G}_{2}\left(\zeta_{2}\right)=\zeta_{2}-a_{3}\left(a_{1}-a_{2} \partial_{X}^{2}\right)^{-1} \zeta^{\star 2} \zeta_{2}
\end{aligned}
$$

and

$$
\begin{aligned}
& H_{\mathrm{e}}^{1}(\mathbb{R}):=\left\{u \in H^{1}(\mathbb{R}): u(X)=u(-X) \text { for all } X \in \mathbb{R}\right\} \\
& H_{\mathrm{o}}^{1}(\mathbb{R}):=\left\{u \in H^{1}(\mathbb{R}): u(X)=-u(-X) \text { for all } X \in \mathbb{R}\right\}
\end{aligned}
$$

Proposition 5.8 The formulae

$$
\zeta_{1} \mapsto-3 a_{3}\left(a_{1}-a_{2} \partial_{X}^{2}\right)^{-1} \zeta^{\star 2} \zeta_{1}, \quad \zeta_{2} \mapsto-a_{3}\left(a_{1}-a_{2} \partial_{X}^{2}\right)^{-1} \zeta^{\star 2} \zeta_{2}
$$

define compact linear operators $H^{1}(\mathbb{R}) \rightarrow H^{1}(\mathbb{R}), H_{\mathrm{e}}^{1}(\mathbb{R}) \rightarrow H_{\mathrm{e}}^{1}(\mathbb{R})$ and $H_{\mathrm{o}}^{1}(\mathbb{R}) \rightarrow H_{\mathrm{o}}^{1}(\mathbb{R})$.

The previous proposition implies in particular that $\mathcal{G}_{1}, \mathcal{G}_{2}$ are Fredholm operators with index 0 . The kernel of $\mathcal{G}_{1}$ coincides with the set of symmetric bounded solutions of the ordinary differential equation

$$
-a_{1} \zeta_{1 X X}+a_{2} \zeta_{1}-3 a_{3} \zeta^{\star 2} \zeta_{1}=0
$$

while the kernel of $\mathcal{G}_{2}$ coincides with the set of antisymmetric bounded solutions of the ordinary differential equation

$$
-a_{1} \zeta_{1 X X}+a_{2} \zeta_{1}-a_{3} \zeta^{\star 2} \zeta_{1}=0
$$

and the next proposition shows that these sets consists of only the trivial solution, so that $\mathcal{G}_{1}, \mathcal{G}_{2}$ and hence $\mathrm{d}_{1} \mathcal{G}\left[\zeta^{\star}, 0\right]$ are isomorphisms.

\section{Proposition 5.9}

(i) Every bounded solution to the equation (5.7) is a multiple of $\zeta_{X}^{\star}$ and is therefore antisymmetric.

(ii) Every bounded solution to the equation (5.8) is a multiple of $\zeta^{\star}$ and is therefore symmetric. 
Proof Introducing the scaled variables

$$
\check{\zeta}_{1}(\check{X})=\left(a_{3} / a_{2}\right)^{1 / 2} \zeta_{1}(X), \quad \check{\zeta}_{2}(\check{X})=\left(a_{3} / a_{2}\right)^{1 / 2} \zeta_{2}(X), \quad \check{X}=\left(a_{2} / a_{1}\right)^{1 / 2} X
$$

transforms Eqs. (5.7), (5.8) into

$$
\begin{aligned}
& -\check{\zeta}_{1 \check{X} \check{X}}+\check{\zeta}_{1}-3 \check{\zeta}^{\star 2} \check{\zeta}_{1}=0, \\
& -\check{\zeta}_{2 \check{X} \check{X}}+\check{\zeta}_{2}-\check{\zeta}^{\star 2} \check{\zeta}_{2}=0,
\end{aligned}
$$

where $\check{\zeta}^{\star}(\check{X})=\sqrt{2}$ sech $\check{X}$, and it obviously suffices to establish the corresponding results for these equations.

Define

$$
\begin{aligned}
& \check{\zeta}_{1,1}(\check{X})=\operatorname{sech} \check{X} \tanh \check{X}, \\
& \check{\zeta}_{1,2}(\check{X})=\operatorname{sech} \check{X}\left(-3+\cosh ^{2} \check{X}+3 \check{X} \tanh \check{X}\right), \\
& \check{\zeta}_{2,1}(\check{X})=\operatorname{sech} \check{X}, \\
& \check{\zeta}_{2,2}(\check{X})=\operatorname{sech} \check{X}(2 \check{X}+\sinh 2 \check{X})
\end{aligned}
$$

and observe that $\left\{\zeta_{1,1}, \zeta_{1,2}\right\}$ is a fundamental solution set for (5.9), whose bounded solutions are therefore precisely the multiplies of $\check{\zeta}_{\check{X}}^{\star}$, while $\left\{\zeta_{2,1}, \zeta_{2,2}\right\}$ is a fundamental solution set for (5.10), whose bounded solutions are therefore precisely the multiples of $\check{\zeta}^{\star}$.

Funding Open Access funding enabled and organised by Projekt DEAL.

Open Access This article is licensed under a Creative Commons Attribution 4.0 International License, which permits use, sharing, adaptation, distribution and reproduction in any medium or format, as long as you give appropriate credit to the original author(s) and the source, provide a link to the Creative Commons licence, and indicate if changes were made. The images or other third party material in this article are included in the article's Creative Commons licence, unless indicated otherwise in a credit line to the material. If material is not included in the article's Creative Commons licence and your intended use is not permitted by statutory regulation or exceeds the permitted use, you will need to obtain permission directly from the copyright holder. To view a copy of this licence, visit http://creativecommons.org/licenses/by/4.0/.

\section{References}

1. Ablowitz, M.J., Segur, H.: On the evolution of packets of water waves. J. Fluid Mech. 92, 691-715 (1979)

2. Amick, C.J., Kirchgässner, K.: A theory of solitary water waves in the presence of surface tension. Arch. Rat. Mech. Anal. 105, 1-49 (1989)

3. Benjamin, T.B.: The solitary wave with surface tension. Q. Appl. Math. 40, 231-234 (1982)

4. Buffoni, B.: Existence and conditional energetic stability of capillary-gravity solitary water waves by minimisation. Arch. Rat. Mech. Anal. 173, 25-68 (2004)

5. Buffoni, B.: Conditional energetic stability of gravity solitary waves in the presence of weak surface tension. Topol. Meth. Nonlinear Anal. 25, 41-68 (2005) 
6. Buffoni, B., Toland, J.F.: Analytic theory of global bifurcation. Princeton University Press, Princeton (2003)

7. Craig, W., Sulem, C.: Numerical simulation of gravity waves. J. Comp. Phys. 108, 73-83 (1993)

8. Dias, F., Kharif, C.: Nonlinear gravity and capillary-gravity waves. Ann. Rev. Fluid Mech. 31, 301-346 (1999)

9. Ehrnström, M., Groves, M.D., Wahlén, E.: On the existence and stability of solitary-wave solutions to a class of evolution equations of Whitham type. Nonlinearity 25, 2903-2936 (2012)

10. Friesecke, G., Pego, R.L.: Solitary waves on FPU lattices: I. Qualitative properties, renormalization and continuum limit. Nonlinearity 12, 1601-1627 (1999)

11. Groves, M.D., Wahlén, E.: On the existence and conditional energetic stability of solitary water waves with weak surface tension. C. R. Math. Acad. Sci. Paris 348, 397-402 (2010)

12. Groves, M.D., Wahlén, E.: Existence and conditional energetic stability of solitary gravity-capillary water waves with constant vorticity. Proc. R. Soc. Edin. A 145, 791-883 (2015)

13. Hörmander, L.: Lectures on nonlinear hyperbolic differential equations. Springer, Heidelberg (1997)

14. Hu, B., Nicholls, D.P.: Analyticity of Dirichlet-Neumann operators on Hölder and Lipschitz domains. SIAM J. Math. Anal. 37, 302-320 (2006)

15. Iooss, G., Kirchgässner, K.: Bifurcation d'ondes solitaires en présence d'une faible tension superficielle. C. R. Acad. Sci. Paris, Sér. 1 311, 265-268 (1990)

16. Iooss, G., Pérouème, M.C.: Perturbed homoclinic solutions in reversible 1:1 resonance vector fields. J. Diff. Equ. 102, 62-88 (1993)

17. Kirchgässner, K.: Nonlinearly resonant surface waves and homoclinic bifurcation. Adv. Appl. Mech. 26, 135-181 (1988)

18. Lannes, D.: The water waves problem: mathematical analysis and asymptotics. Number 188 in Mathematical surveys and monographs. American Mathematical Society, Providence (2013)

19. Nicholls, D.P., Reitich, F.: A new approach to analyticity of Dirichlet-Neumann operators. Proc. R. Soc. Edin. A 131, 1411-1433 (2001)

20. Sachs, R.L.: On the existence of small amplitude solitary waves with strong surface-tension. J. Diff. Equ. 90, 31-51 (1991)

21. Stefanov, A., Wright, J.D.: Small amplitude traveling waves in the full-dispersion Whitham equation. J. Dyn. Diff. Equ. 32, 85-99 (2020)

22. Zakharov, V.E.: Stability of periodic waves of finite amplitude on the surface of a deep fluid. Zh. Prikl. Mekh. Tekh. Fiz. 9, 86-94 (1968). (English translation J. Appl. Mech. Tech. Phys. 9, 190-194.)

Publisher's Note Springer Nature remains neutral with regard to jurisdictional claims in published maps and institutional affiliations. 